\title{
Sharp Constants of Brézis-Gallouët-Wainger Type Inequalities with a Double Logarithmic Term on Bounded Domains in Besov and Triebel-Lizorkin Spaces
}

\author{
Kei Morii, ${ }^{1}$ Tokushi Sato, ${ }^{2}$ Yoshihiro Sawano, $^{3}$ \\ and Hidemitsu Wadade ${ }^{4}$ \\ ${ }^{1}$ Heian Jogakuin St. Agnes' School, 172-2, Gochomecho, Kamigyo-ku, Kyoto 602-8013, Japan \\ ${ }^{2}$ Mathematical Institute, Tohoku University, Sendai 980-8578, Japan \\ ${ }^{3}$ Department of Mathematics, Kyoto University, Kyoto 606-8502, Japan \\ ${ }^{4}$ Department of Mathematics, Osaka City University, 3-3-138 Sugimoto, Sumiyoshi-ku, \\ Osaka 558-8585, Japan \\ Correspondence should be addressed to Yoshihiro Sawano, yoshihiro-sawano@celery.ocn.ne.jp
}

Received 4 October 2009; Revised 15 September 2010; Accepted 12 October 2010

Academic Editor: Veli B. Shakhmurov

Copyright (C) 2010 Kei Morii et al. This is an open access article distributed under the Creative Commons Attribution License, which permits unrestricted use, distribution, and reproduction in any medium, provided the original work is properly cited.

The present paper concerns the Sobolev embedding in the endpoint case. It is known that the embedding $W^{1, n}\left(\mathbb{R}^{n}\right) \hookrightarrow L^{\infty}\left(\mathbb{R}^{n}\right)$ fails for $n \geq 2$. Brézis-Gallouët-Wainger and some other authors quantified why this embedding fails by means of the Hölder-Zygmund norm. In the present paper we will give a complete quantification of their results and clarify the sharp constants for the coefficients of the logarithmic terms in Besov and Triebel-Lizorkin spaces.

\section{Introduction and Known Results}

We establish sharp Brézis-Gallouët-Wainger type inequalities in Besov and Triebel-Lizorkin spaces as well as fractional Sobolev spaces on a bounded domain $\Omega \subset \mathbb{R}^{n}$. Throughout the present paper, we place ourselves in the setting of $\mathbb{R}^{n}$ with $n \geq 2$. We treat only real-valued functions.

First we recall the Sobolev embedding theorem in the critical case. For $1<q<\infty$, it is well known that the embedding $W^{n / q, q}\left(\mathbb{R}^{n}\right) \hookrightarrow L^{r}\left(\mathbb{R}^{n}\right)$ holds for any $q \leq r<\infty$, and does not hold for $r=\infty$, that is, one cannot estimate the $L^{\infty}$-norm by the $W^{n / q, q}$-norm. However, the 
Brézis-Gallouët-Wainger inequality states that the $L^{\infty}$-norm can be estimated by the $W^{n / q, q_{-}}$ norm with the partial aid of the $W^{s, p}$-norm with $s>n / p$ and $1 \leq p \leq \infty$ as follows:

$$
\|u\|_{L^{\infty}\left(\mathbb{R}^{n}\right)}^{q /(q-1)} \leq \lambda\left(1+\log \left(1+\|u\|_{W^{s, p}\left(\mathbb{R}^{n}\right)}\right)\right)
$$

holds whenever $u \in W^{n / q, q}\left(\mathbb{R}^{n}\right) \cap W^{s, p}\left(\mathbb{R}^{n}\right)$ satisfies $\|u\|_{W^{n / q, q\left(\mathbb{R}^{n}\right)}}=1$, where $1 \leq p \leq \infty$, $1<q<\infty$, and $s>n / p$. Inequality (1.1) for the case $n=p=q=s=2$ dates back to Brézis-Gallouët [1]. Later on, Brézis and Wainger [2] obtained (1.1) for the general case, and remarked that the power $q /(q-1)$ in (1.1) is maximal; equation (1.1) fails for any larger power. Ozawa [3] proved (1.1) with the Sobolev norm $\|u\|_{W^{s, p}\left(\mathbb{R}^{n}\right)}$ in (1.1) replaced by the homogeneous Sobolev norm $\|u\|_{\dot{W}^{s, p}\left(\mathbb{R}^{n}\right)}$. An attempt of replacing $\|u\|_{W^{s, p}\left(\mathbb{R}^{n}\right)}$ with the other norms has been made in several papers. For instance, Kozono et al. [4] generalized (1.1) with both of $W^{n / q, q}\left(\mathbb{R}^{n}\right)$ and $W^{s, p}\left(\mathbb{R}^{n}\right)$ replaced by the Besov spaces and applied it to the regularity problem for the Navier-Stokes equation and the Euler equation. Moreover, Ogawa [5] proved (1.1) in terms of Triebel-Lizorkin spaces for the purpose to investigate the regularity to the gradient flow of the harmonic map into a sphere. We also mention that (1.1) was obtained in the Besov-Morrey spaces in [6].

In what follows, we concentrate on the case $q=n$ and replace the function space $W^{n / q, q}\left(\mathbb{R}^{n}\right)$ by $W_{0}^{1, n}(\Omega)$ with a bounded domain $\Omega$ in $\mathbb{R}^{n}$. Note that the norm of $W_{0}^{1, n}(\Omega)$ is equivalent to $\|\nabla u\|_{L^{n}(\Omega)}$ because of the Poincaré inequality. When the differential order $s=m$ is an integer with $1 \leq m \leq n$, and $n / m<p \leq n /(m-1)$, the first, second and fourth authors [7] generalized the inequality corresponding to (1.1) and discussed how optimal the constant $\lambda$ is. To describe the sharpness of the constant $\lambda$, they made a formulation more precise as follows:

For given constants $\lambda_{1}>0$ and $\lambda_{2} \in \mathbb{R}$, does there exist a constant $C$ such that

$$
\begin{aligned}
\|u\|_{L^{\infty}(\Omega)}^{n /(n-1)} \leq & \lambda_{1} \log \left(1+\|u\|_{W^{s, p}(\Omega)}\right) \\
& +\lambda_{2} \log \left(1+\log \left(1+\|u\|_{W^{s, p}(\Omega)}\right)\right)+C
\end{aligned}
$$

holds for all $u \in W_{0}^{1, n}(\Omega) \cap W^{s, p}(\Omega)$ with $\|\nabla u\|_{L^{n}(\Omega)}=1$ ?

Here for the sake of definiteness, define

$$
\|\nabla u\|_{L^{n}(\Omega)}=\left\|\left|\nabla u \|_{L^{n}(\Omega)}, \quad\right| \nabla u \mid=\left(\sum_{i=1}^{n}\left|\frac{\partial u}{\partial x_{i}}\right|^{2}\right)^{1 / 2} .\right.
$$

We call the first term and the second term of the right-hand side of (1.2) the single logarithmic term and the double logarithmic term, respectively. We remark that the double logarithmic term grows weaker than the single one as $\|u\|_{W^{s, p}(\Omega)} \rightarrow \infty$.

Then they proved the following theorem, which gives the sharp constants for $\lambda_{1}$ and $\lambda_{2}$ in (1.2). Here and below, $\Lambda_{1}$ and $\Lambda_{2}$ are constants defined by

$$
\Lambda_{1}=\frac{1}{\omega_{n-1}^{1 /(n-1)}}, \quad \Lambda_{2}=\frac{\Lambda_{1}}{n}=\frac{1}{n \omega_{n-1}^{1 /(n-1)}},
$$


where $\omega_{n-1}=2 \pi^{n / 2} / \Gamma(n / 2)$ is the surface area of $S^{n-1}=\left\{x \in \mathbb{R}^{n} ;|x|=1\right\}$. See Definition 2.5 below for the definition of the strong local Lipschitz condition for a domain $\Omega$.

Theorem 1.1 ([7, Theorem 1.2]). Let $n \geq 2,0<\alpha<1, m \in\{1,2, \ldots, n\}$, and, $\Omega$ be a bounded domain in $\mathbb{R}^{n}$ satisfying the strong local Lipschitz condition.

(i) Assume that either

$$
\text { (I) } \lambda_{1}>\frac{\Lambda_{1}}{\alpha}, \quad \lambda_{2} \in \mathbb{R} \quad \text { or } \quad \text { (II) } \lambda_{1}=\frac{\Lambda_{1}}{\alpha}, \quad \lambda_{2} \geq \frac{\Lambda_{2}}{\alpha}
$$

holds. Then there exists a constant $C$ such that inequality (1.2) with $s=m$ and $p=$ $n /(m-\alpha)$ holds for all $u \in W_{0}^{1, n}(\Omega) \cap W^{m, n /(m-\alpha)}(\Omega)$ with $\|\nabla u\|_{L^{n}(\Omega)}=1$.

(ii) Assume that either

$$
\text { (III) } \lambda_{1}<\frac{\Lambda_{1}}{\alpha}, \quad \lambda_{2} \in \mathbb{R} \quad \text { or } \quad(I V) \lambda_{1}=\frac{\Lambda_{1}}{\alpha}, \quad \lambda_{2}<\frac{\Lambda_{2}}{\alpha}
$$

holds. Then for any constant $C$, inequality (1.2) with $s=m$ and $p=n /(m-\alpha)$ fails for some $u \in W_{0}^{1, n}(\Omega) \cap W_{0}^{m, n /(m-\alpha)}(\Omega)$ with $\|\nabla u\|_{L^{n}(\Omega)}=1$.

We note that the differential order $m$ of the higher order Sobolev space in Theorem 1.1 had to be an integer. The primary aim of the present paper is to pass Theorem 1.1 to those which include Sobolev spaces of fractional differential order. Meanwhile, higher-order Sobolev spaces are continuously embedded into corresponding Hölder spaces. Standing on such a viewpoint, the first, second, and fourth authors [8] proved a result similar to Theorem 1.1 for the homogeneous Hölder space $\dot{C}^{0, \alpha}(\Omega)$ instead of the Sobolev space $W^{m, n /(m-\alpha)}(\Omega)$. Furthermore, it is known that the Hölder space $C^{0, \alpha}(\Omega)$ is expressed as the marginal case of the Besov space $B^{\alpha, \infty, \infty}(\Omega)$ provided that $0<\alpha<1$, which allows us to extend Theorem 1.1 with the same sharp constants in Besov spaces.

In general, we set up the following problem in a fixed function space $X(\Omega)$, which is contained in $L^{\infty}(\Omega)$.

Fix a function space $X(\Omega)$. For given constants $\lambda_{1}>0$ and $\lambda_{2} \in \mathbb{R}$, does there exist a constant $C$ such that

$$
\begin{aligned}
\|u\|_{L^{\infty}(\Omega)}^{n /(n-1)} \leq & \lambda_{1} \log \left(1+\|u\|_{X(\Omega)}\right) \\
& +\lambda_{2} \log \left(1+\log \left(1+\|u\|_{X(\Omega)}\right)\right)+C
\end{aligned}
$$

holds for all $u \in W_{0}^{1, n}(\Omega) \cap X(\Omega)$ under the normalization $\|\nabla u\|_{L^{n}(\Omega)}=1$ ?

We call $W^{s, p}\left(\mathbb{R}^{n}\right)$ an auxiliary space of (1.7). First we state the following proposition, which is an immediate consequence of an elementary inequality,

$$
\log (1+s t) \leq \log (s+s t)=\log (1+t)+\log s \quad \text { for } t \geq 0, \quad s \geq 1 .
$$


Proposition 1.2. Let $\Omega$ be a domain in $\mathbb{R}^{n}$, and let $X_{1}(\Omega), X_{2}(\Omega)$ be function spaces satisfying

$$
\|u\|_{X_{1}(\Omega)} \leq M\|u\|_{X_{2}(\Omega)} \quad \text { for } u \in X_{2}(\Omega)
$$

with some constant $M \geq 1$.

(i) If inequality (1.7) holds in $X(\Omega)=X_{1}(\Omega)$ with a constant $C$, then so does $(1.7)$ in $X(\Omega)=$ $X_{2}(\Omega)$ with another constant $C$,

or equivalently,

(i) if inequality (1.7) fails in $X(\Omega)=X_{2}(\Omega)$ with any constant $C$, then so does (1.7) in $X(\Omega)=X_{1}(\Omega)$ with any constant $C$.

From the proposition above, the sharp constants for $\lambda_{1}$ and $\lambda_{2}$ in (1.7) are independent of the choice of the equivalent norms of the auxiliary space $X(\Omega)$. On the other hand, note that these sharp constants may depend on the definition of $\|\nabla u\|_{L^{n}(\Omega)}$; there are several manners to define $\|\nabla u\|_{L^{n}(\Omega)}$. In what follows, we choose (1.3) as the definition of $\|\nabla u\|_{L^{n}(\Omega)}$.

In the present paper we will include Besov and Triebel-Lizorkin spaces as an auxiliary space $X(\Omega)$. To describe the definition of Besov and Triebel-Lizorkin spaces, we denote by $B_{R}$ the open ball in $\mathbb{R}^{n}$ centered at the origin with radius $R>0$, that is, $B_{R}=\left\{x \in \mathbb{R}^{n} ;|x|<R\right\}$. Define the Fourier transform $\mathcal{F}$ and its inverse $\mathcal{F}^{-1}$ by

$$
\mathcal{F} u(\xi)=\frac{1}{(2 \pi)^{n / 2}} \int_{\mathbb{R}^{n}} e^{-\sqrt{-1} x \cdot \xi} u(x) d x, \quad \mathcal{F}^{-1} u(x)=\frac{1}{(2 \pi)^{n / 2}} \int_{\mathbb{R}^{n}} e^{\sqrt{-1} x \cdot \xi} u(\xi) d \xi
$$

for $u \in \mathcal{S}\left(\mathbb{R}^{n}\right)$, respectively, and they are also extended on $\mathcal{S}^{\prime}\left(\mathbb{R}^{n}\right)$ by the usual way. For $\varphi \in \mathcal{S}\left(\mathbb{R}^{n}\right)$, define an operator $\varphi(D)$ by

$$
\varphi(D) u=\mathcal{F}^{-1}(\varphi \mathscr{F} u)=\frac{1}{(2 \pi)^{n / 2}}\left(\mathcal{F}^{-1} \varphi\right) * u
$$

Next, we fix functions $\psi^{0}, \varphi^{0} \in C_{\mathrm{c}}^{\infty}\left(\mathbb{R}^{n}\right)$ which are supported in the ball $\bar{B}_{4}$, in the annulus $\bar{B}_{4} \backslash B_{1}$, respectively, and satisfying

$$
\sum_{k=-\infty}^{\infty} \varphi_{k}^{0}(x)=\chi_{\mathbb{R}^{n} \backslash\{0\}}(x), \quad \psi^{0}(x)=1-\sum_{k=0}^{\infty} \varphi_{k}^{0}(x) \quad \text { for } x \in \mathbb{R}^{n},
$$

where we set $\varphi_{k}^{0}=\varphi^{0}\left(\cdot / 2^{k}\right)$. Here, $\chi_{E}$ is the characteristic function of a set $E$ and $C_{\mathrm{c}}^{\infty}(\Omega)$ denotes the class of compactly supported $C^{\infty}$-functions on $\Omega$. We also denote by $C_{\mathrm{c}}(\Omega)$ the class of compactly supported continuous functions on $\Omega$.

Definition 1.3. Take $\psi^{0}, \varphi^{0}$ satisfying (1.12), and let $u \in \mathcal{S}^{\prime}\left(\mathbb{R}^{n}\right)$.

(i) Let $0<s<\infty, 0<p \leq \infty$, and $0<q \leq \infty$. The Besov space $B^{s, p, q}\left(\mathbb{R}^{n}\right)$ is normed by

$$
\|u\|_{B^{s, p, q\left(\mathbb{R}^{n}\right)}}=\left\|\psi^{0}(D) u\right\|_{L^{p}\left(\mathbb{R}^{n}\right)}+\left(\sum_{k=0}^{\infty}\left(2^{s k}\left\|\varphi_{k}^{0}(D) u\right\|_{L^{p}\left(\mathbb{R}^{n}\right)}\right)^{q}\right)^{1 / q}
$$


with the obvious modification when $q=\infty$.

(ii) Let $0<s<\infty, 0<p<\infty$, and $0<q \leq \infty$. The Triebel-Lizorkin space $F^{s, p, q}\left(\mathbb{R}^{n}\right)$ is normed by

$$
\|u\|_{F^{s, p, q\left(\mathbb{R}^{n}\right)}}=\left\|\psi^{0}(D) u\right\|_{L^{p}\left(\mathbb{R}^{n}\right)}+\left\|\left(\sum_{k=0}^{\infty}\left(2^{s k}\left|\varphi_{k}^{0}(D) u\right|\right)^{q}\right)^{1 / q}\right\|_{L^{p}\left(\mathbb{R}^{n}\right)}
$$

with the obvious modification when $q=\infty$; one excludes the case $p=\infty$.

Different choices of $\psi^{0}$ and $\varphi^{0}$ satisfying (1.12) yield equivalent norms in (1.13) and (1.14). We refer to [9] for exhaustive details of this fact. Here and below, we denote by $A^{s, p, q}$ the spaces $B^{s, p, q}$ with $0<s<\infty, 0<p \leq \infty, 0<q \leq \infty$, or $F^{s, p, q}$ with $0<s<\infty, 0<p<\infty, 0<$ $q \leq \infty$. Unless otherwise stated, the letter $A$ means the same scale throughout the statement.

As in $[9,10]$, we adopt a traditional method of defining function spaces on a domain $\Omega \subset \mathbb{R}^{n}$.

Definition 1.4. Let $0<s<\infty$ and $0<p, q \leq \infty$.

(i) The function space $A^{s, p, q}(\Omega)$ is defined as the subset of $\Phi^{\prime}(\Omega)$ obtained by restricting elements in $A^{s, p, q}\left(\mathbb{R}^{n}\right)$ to $\Omega$, and the norm is given by

$$
\|u\|_{A^{s, p, q}(\Omega)}=\inf \left\{\|v\|_{A^{s, p, q\left(\mathbb{R}^{n}\right)}} ; v \in A^{s, p, q}\left(\mathbb{R}^{n}\right),\left.v\right|_{\Omega}=u \text { in } \Phi^{\prime}(\Omega)\right\}
$$

(ii) The function space $A_{0}^{s, p, q}(\Omega)$ is defined as the closure of $C_{c}^{\infty}(\Omega)$ in the norm of $A^{s, p, q}(\Omega)$.

(iii) The potential space $H^{s, p}(\Omega)$ stands for $F^{s, p, 2}(\Omega)$.

Now we state our main result, which claims that the sharp constants in (1.7) are given by the same ones as in Theorem 1.1 when $X(\Omega)=A^{s, p_{\alpha, s}, q}(\Omega)$ or $A_{0}^{s, p_{\alpha, s}, q}(\Omega)$, where in what follows we denote

$$
p_{\alpha, s}= \begin{cases}\frac{n}{s-\alpha} & \text { for } s>\alpha \\ \infty & \text { for } s=\alpha\end{cases}
$$

Here, conditions (I)-(IV) are the same as in Theorem 1.1. We should remark that $A_{0}^{s, p_{\alpha, s}, q}(\Omega) \subset$ $A^{s, p_{\alpha, s}, q}(\Omega) \subset L^{\infty}(\Omega)$ and the formulation of Theorem 1.5 remains unchanged no matter what equivalent norms we choose for the norm of the function space $A^{s, p_{\alpha, s}, q}(\Omega)$. Indeed, Proposition 1.2(i) (resp., (ii)) shows that the condition on $\lambda_{1}$ and $\lambda_{2}$ for which inequality (1.7) holds (resp., fails) remains unchanged if we replace the definition of the norm $\|\cdot\|_{A^{s, p a, s, q}(\Omega)}$ with any equivalent norm.

In the case $0<\alpha<1$, we can determine the condition completely.

Theorem 1.5. Let $n \geq 2,0<\alpha<1, s \geq \alpha, 0<q \leq \infty$, and let $\Omega$ be a bounded domain in $\mathbb{R}^{n}$ and $X(\Omega)=A^{s, p_{\alpha, s}, q}(\Omega)$. 
(i) Assume that either (I) or (II) holds. Then there exists a constant $C$ such that inequality (1.7) holds for all $u \in W_{0}^{1, n}(\Omega) \cap A^{s, p_{\alpha, s}, q}(\Omega)$ with $\|\nabla u\|_{L^{n}(\Omega)}=1$.

(ii) Assume that either (III) or (IV) holds. Then for any constant $C$, the inequality (1.7) fails for some $u \in C_{c}^{\infty}(\Omega)$ with $\|\nabla u\|_{L^{n}(\Omega)}=1$.

Remark 1.6. If $\Omega$ has a Lipschitz boundary, then the Stein total extension theorem [11, Theorem 5.24] implies that $W^{m, p}(\Omega)=H^{m, p}(\Omega)=F^{m, p, 2}(\Omega)$ for $m \in \mathbb{N}$ and $1<p<\infty$. Hence Theorem 1.5 implies Theorem 1.1.

In order to state our results in the case $\alpha \geq 1$ for a general bounded domain $\Omega$, we replace assumption (II) by the slightly stronger one

$$
(\text { II })^{\prime} \lambda_{1}=\frac{\Lambda_{1}}{\alpha}, \quad \lambda_{2}>\Lambda_{2}
$$

Unfortunately, we do not know whether the result in this case corresponding to the case $\alpha \geq 1$ in Theorem 1.5 holds.

Theorem 1.7. Let $n \geq 2, \alpha \geq 1, s \geq \alpha, 0<q \leq \infty$, let $\Omega$ be a bounded domain in $\mathbb{R}^{n}$ satisfying the strong local Lipschitz condition and $X(\Omega)=A^{s, p_{\alpha, s}, q}(\Omega)$.

(i) Assume that either (I) or (II)' holds. Then there exists a constant $C$ such that inequality (1.7) holds for all $u \in W_{0}^{1, n}(\Omega) \cap A^{s, p_{\alpha, s}, q}(\Omega)$ with $\|\nabla u\|_{L^{n}(\Omega)}=1$.

(ii) Assume that either (III) or (IV) holds. Then for any constant $C$, the inequality (1.7) fails for some $u \in C_{c}^{\infty}(\Omega)$ with $\|\nabla u\|_{L^{n}(\Omega)}=1$.

Remark 1.8. We have to impose the strong local Lipschitz condition in Theorem 1.7, because we use the universal extension theorem obtained by Rychkov [12, Theorem 2.2].

However, in the case $1<\alpha<2$, we can also determine the condition completely as in the case $0<\alpha<1$ provided that we restrict the functions to $C_{c}(\Omega)$.

Theorem 1.9. Let $n \geq 2,1<\alpha<2, s \geq \alpha, 0<q \leq \infty$, let $\Omega$ be a bounded domain in $\mathbb{R}^{n}$, and $X(\Omega)=A^{s, p_{\alpha, s}, q}(\Omega)$.

(i) Assume that either (I) or (II) holds. Then there exists a constant $C$ such that inequality (1.7) holds for all $u \in W_{0}^{1, n}(\Omega) \cap A^{s, p_{\alpha, s}, q}(\Omega) \cap C_{c}(\Omega)$ with $\|\nabla u\|_{L^{n}(\Omega)}=1$.

(ii) Assume that either (III) or (IV) holds. Then for any constant $C$, inequality (1.7) fails for some $u \in C_{c}^{\infty}(\Omega)$ with $\|\nabla u\|_{L^{n}(\Omega)}=1$.

We also obtain the following corollary because $C_{c}^{\infty}(\Omega) \subset A_{0}^{s, p, q}(\Omega) \subset A^{s, p, q}(\Omega)$.

Corollary 1.10. Theorems $1.5,1.7$, and 1.9 still hold true if one replaces $A^{s, p_{\alpha, s}, q}(\Omega)$ by $A_{0}^{s, p_{\alpha, s}, q}(\Omega)$.

Remark 1.11. (i) The assertion in Corollary 1.10 corresponding to Theorem 1.7 still holds even if we do not impose the strong local Lipschitz condition, because there is a trivial extension operator from $A_{0}^{s, p, q}(\Omega)$ into $A^{s, p, q}\left(\mathbb{R}^{n}\right)$.

(ii) If $\partial \Omega$ is smooth, then we can see that

$$
u \in C(\bar{\Omega}), \quad u=0 \quad \text { on } \partial \Omega \quad \text { for } u \in W_{0}^{1, n}(\Omega) \cap A^{s, p_{\alpha, s}, q}(\Omega) \text {. }
$$


However, $W_{0}^{1, n}(\Omega) \cap A^{s, p_{\alpha, s}, q}(\Omega)$ is not contained in $A_{0}^{s, p_{\alpha, s}, q}(\Omega)$, in general.

Remark 1.12. The power $n /(n-1)$ on the left-hand side of (1.7) is optimal in the sense that $r=n /(n-1)$ is the largest power for which there exist $\lambda_{1}$ and $C$ such that

$$
\|u\|_{L^{\infty}(\Omega)}^{r} \leq \lambda_{1} \log \left(1+\|u\|_{X(\Omega)}\right)+C
$$

can hold for all $u \in W_{0}^{1, n}(\Omega) \cap X(\Omega)$ with $\|\nabla u\|_{L^{n}(\Omega)}=1$. Here, $X(\Omega)$ is as in Theorems 1.5, 1.7, and 1.9 and Corollary 1.10. Indeed, if $r>n /(n-1)$, then for any $\lambda_{1}>0$ and any constant $C$, (1.19) does not hold for some $u \in W_{0}^{1, n}(\Omega) \cap X(\Omega)$ with $\|\nabla u\|_{L^{n}(\Omega)}=1$, which is shown by carrying out a similar calculation to the proof of Theorems 1.5, 1.7, and 1.9 (ii); see Remark 3.9 below for the details. To the contrary, if $1 \leq r<n /(n-1)$, then for any $\lambda_{1}>0$, there exists a constant $C$ such that (1.19) holds for all $u \in W_{0}^{1, n}(\Omega) \cap X(\Omega)$ with $\|\nabla u\|_{L^{n}(\Omega)}=1$. This fact follows from the embedding described below and the same assertion concerning the BrézisGallouët-Wainger type inequality in the Hölder space, which is shown in [8, Remark 3.5] (for $0<\alpha<1$ ) and Remark 4.3 (for $\alpha \geq 1$ ).

Finally let us describe the organization of the present paper. In Section 2, we introduce some notation of function spaces and state embedding theorems. Section 3 is devoted to proving the negative assertions of Theorems 1.5-1.9. Section 4 describes the affirmative assertions of Theorems 1.5 and 1.7. Section 5 concerns the affirmative assertion of Theorem 1.9. In the appendix, we prove elementary calculus which we stated in Section 5.

\section{Preliminaries}

First we provide a brief view of Hölder and Hölder-Zygmund spaces. Throughout the present paper, $C$ denotes a constant which may vary from line to line.

For $0<\alpha \leq 1, \dot{C}^{0, \alpha}\left(\mathbb{R}^{n}\right)$ denotes the homogeneous Hölder space of order $\alpha$ endowed with the seminorm

$$
\|u\|_{\dot{C}^{0, \alpha}\left(\mathbb{R}^{n}\right)}=\sup _{\substack{x, y \in \mathbb{R}^{n} \\ x \neq y}} \frac{|u(x)-u(y)|}{|x-y|^{\alpha}},
$$

and $C^{0, \alpha}\left(\mathbb{R}^{n}\right)$ denotes the nonhomogeneous Hölder space of order $\alpha$ endowed with the norm

$$
\|u\|_{C^{0, \alpha}\left(\mathbb{R}^{n}\right)}=\|u\|_{L^{\infty}\left(\mathbb{R}^{n}\right)}+\|u\|_{\dot{C}^{0, \alpha}\left(\mathbb{R}^{n}\right)} .
$$

Define also

$$
\|\mathbf{u}\|_{\dot{C}^{0, \alpha}\left(\mathbb{R}^{n} ; \mathbb{R}^{n}\right)}=\sup _{\substack{x, y \in \mathbb{R}^{n} \\ x \neq y}} \frac{|\mathbf{u}(x)-\mathbf{u}(y)|}{|x-y|^{\alpha}}
$$


for an $\mathbb{R}^{n}$-valued function $\mathbf{u}$. For $1 \leq \alpha \leq 2, \dot{C}^{1, \alpha-1}\left(\mathbb{R}^{n}\right)$ denotes the homogeneous HölderZygmund space of order $\alpha$, the set of all continuous functions $u$ endowed with the seminorm

$$
\|u\|_{\dot{C}^{1, \alpha-1}\left(\mathbb{R}^{n}\right)}=\sup _{\substack{x, y \in \mathbb{R}^{n} \\ x \neq y}} \frac{|u(x)-2 u((x+y) / 2)+u(y)|}{|x-y|^{\alpha}}
$$

and $C^{1, \alpha-1}\left(\mathbb{R}^{n}\right)$ denotes the nonhomogeneous Hölder-Zygmund space of order $\alpha$, the set of all continuous functions $u$ endowed with the norm

$$
\|u\|_{C^{1, \alpha-1}\left(\mathbb{R}^{n}\right)}=\|u\|_{L^{\infty}\left(\mathbb{R}^{n}\right)}+\|u\|_{\dot{C}^{1, \alpha-1}\left(\mathbb{R}^{n}\right)} .
$$

Note that $\dot{C}^{0,1}\left(\mathbb{R}^{n}\right)$ is a proper subset of $\dot{C}^{1,0}\left(\mathbb{R}^{n}\right)$. We remark that, in defining $\dot{C}^{1, \alpha-1}\left(\mathbb{R}^{n}\right)$, it is necessary that we assume the functions continuous. Here we will exhibit an example of a discontinuous function $u$ satisfying $\|u\|_{\dot{C}^{1, \alpha-1}\left(\mathbb{R}^{n}\right)}=0$ in the appendix. We will not need to define the Hölder-Zygmund space of the higher order. We need an auxiliary function space; for $1<\alpha \leq 2$, let $C_{\nabla}^{1, \alpha-1}\left(\mathbb{R}^{n}\right)$ denote the analogue of $C^{1, \alpha-1}\left(\mathbb{R}^{n}\right)$ endowed with the seminorm

$$
\|u\|_{\dot{C}_{\nabla}^{1, \alpha-1}\left(\mathbb{R}^{n}\right)}=\|\nabla u\|_{\dot{C}^{0, \alpha-1}\left(\mathbb{R}^{n} ; \mathbb{R}^{n}\right)}=\sup _{\substack{x, y \in \mathbb{R}^{n} \\ x \neq y}} \frac{|\nabla u(x)-\nabla u(y)|}{|x-y|^{\alpha-1}} .
$$

The other function spaces on a domain $\Omega \subset \mathbb{R}^{n}$ are made analogously to $A^{s, p, q}(\Omega)$. For example, define

$$
\begin{gathered}
\|u\|_{\dot{C}^{0, \alpha}(\Omega)}=\inf \left\{\|v\|_{\dot{C}^{0, \alpha}\left(\mathbb{R}^{n}\right)} ; v \in \dot{C}^{0, \alpha}\left(\mathbb{R}^{n}\right),\left.v\right|_{\Omega}=u \text { in } \Phi^{\prime}(\Omega)\right\}, \\
\|u\|_{\dot{C}^{1, \alpha-1}(\Omega)}=\inf \left\{\|v\|_{\dot{C}^{1, \alpha-1}\left(\mathbb{R}^{n}\right)} ; v \in \dot{C}^{1, \alpha-1}\left(\mathbb{R}^{n}\right),\left.v\right|_{\Omega}=u \text { in } \Phi^{\prime}(\Omega)\right\}, \\
\|u\|_{\dot{C}_{\nabla}^{1, \alpha-1}(\Omega)}=\inf \left\{\|\nabla v\|_{\dot{C}^{0, \alpha-1}\left(\mathbb{R}^{n} ; \mathbb{R}^{n}\right)} ; v \in \dot{C}_{\nabla}^{1, \alpha-1}\left(\mathbb{R}^{n}\right),\left.(\nabla v)\right|_{\Omega}=\nabla u \text { in } \boldsymbol{\Phi}^{\prime}(\Omega)\right\} .
\end{gathered}
$$

A moment's reflection shows that for $0<\alpha \leq 1,\|u\|_{\dot{C}^{0, \alpha}(\Omega)}$ can be written as

$$
\|u\|_{C^{0, \alpha}(\Omega)}=\sup _{\substack{x, y \in \Omega \\ x \neq y}} \frac{|u(x)-u(y)|}{|x-y|^{\alpha}} \text { for } u \in \dot{C}^{0, \alpha}(\Omega)
$$

since the function

$$
v(x)=\inf _{y \in \Omega}\left(u(y)+\|u\|_{\dot{C}^{0, \alpha}(\Omega)}|x-y|^{\alpha}\right) \quad \text { for } x \in \mathbb{R}^{n}
$$


attains the infimum defining $\|u\|_{C^{0, \alpha}(\Omega)}$ (see [13, Theorem 3.1.1]). Moreover, we also observe that

$$
\|u\|_{\dot{C}_{\nabla}^{1, \alpha-1}(\Omega)}=\|\nabla u\|_{\dot{C}^{0, \alpha-1}\left(\Omega ; \mathbb{R}^{n}\right)}=\sup _{\substack{x, y \in \Omega \\ x \neq y}} \frac{|\nabla u(x)-\nabla u(y)|}{|x-y|^{\alpha-1}} \text { for } u \in \dot{C}_{\nabla}^{1, \alpha-1}(\Omega) \cap C_{\mathrm{C}}(\Omega)
$$

since the zero-extended function $v$ of $u$ on $\mathbb{R}^{n} \backslash \Omega$ attains the infimum defining $\|\nabla u\|_{\dot{C}^{0, \alpha-1}\left(\Omega ; \mathbb{R}^{n}\right)}$. An elementary relation between these spaces and $B^{\alpha, \infty, \infty}\left(\mathbb{R}^{n}\right)$ is as follows.

Lemma 2.1 (Taibleson, [14, Theorem 4]). Let $0<\alpha<2$. Then one has the norm equivalence

$$
B^{\alpha, \infty, \infty}\left(\mathbb{R}^{n}\right) \simeq C^{\lfloor\alpha\rfloor, \alpha-\lfloor\alpha\rfloor}\left(\mathbb{R}^{n}\right),
$$

where $\lfloor\alpha\rfloor$ denotes the integer part of $\alpha ; \mid \alpha\rfloor=\max \{k \in \mathbb{N} \cup\{0\} ; k \leq \alpha\}$.

We remark that Lemma 2.1 is still valid for $\alpha \geq 2$ after defining the function space $C^{\lfloor\alpha\rfloor, \alpha-\lfloor\alpha\rfloor}\left(\mathbb{R}^{n}\right)$ appropriately. However, we do not go into detail, since we will use the space $C^{\lfloor\alpha\rfloor, \alpha-\lfloor\alpha\rfloor}\left(\mathbb{R}^{n}\right)$ only with $0<\alpha<2$.

We will invoke the following fact on the Sobolev type embedding for Besov and Triebel-Lizorkin spaces:

Lemma 2.2. Let $0<s<\infty, 0<p<\tilde{p} \leq \infty, 0<q<\tilde{q} \leq \infty$, and let $\Omega$ be a domain in $\mathbb{R}^{n}$. Then

$$
\begin{gathered}
B^{s, p, q}(\Omega) \hookrightarrow B^{s, p, \tilde{q}}(\Omega), \\
B^{s, p, q}(\Omega) \hookrightarrow B^{s-n(1 / p-1 / \tilde{p}), \tilde{p}, q}(\Omega), \\
B^{s, p, \min \{p, q\}}(\Omega) \hookrightarrow F^{s, p, q}(\Omega) \hookrightarrow B^{s, p, \max \{p, q\}}(\Omega)
\end{gathered}
$$

in the sense of continuous embedding.

Proof. We accept all the embeddings when $\Omega=\mathbb{R}^{n}$; see [9] for instance. The case when $\Omega$ has smooth boundary is covered in [9]. However, as the proof below shows, the results are still valid even when the boundary of $\Omega$ is not smooth. For the sake of convenience, let us prove the second one. To this end we take $u \in B^{s, p, q}(\Omega)$. Then by the definition of $B^{s, p, q}(\Omega)$ and its norm, we can find $v \in B^{s, p, q}\left(\mathbb{R}^{n}\right)$ so that

$$
\left.v\right|_{\Omega}=u \quad \text { in } \Phi^{\prime}(\Omega), \quad\|u\|_{B^{s, p, q}(\Omega)} \leq\|v\|_{B^{s, p, q}\left(\mathbb{R}^{n}\right)} \leq 2\|u\|_{B^{s, p, q}(\Omega)} .
$$

Now that we accept $\|v\|_{B^{s-n(1 / p-1 / \tilde{p}), \tilde{p}, q}\left(\mathbb{R}^{n}\right)} \leq C_{s, p, \tilde{p}, q}\|v\|_{B^{s, p, q}\left(\mathbb{R}^{n}\right)}$, we have

$$
\|u\|_{B^{s-n(1 / p-1 / \tilde{p}) \tilde{p}, q(\Omega)}} \leq\|v\|_{B^{s-n(1 / p-1 / \tilde{p}), \tilde{p}, q\left(\mathbb{R}^{n}\right)}} \leq C_{s, p, \tilde{p}, q}\|v\|_{B^{s, p, q}\left(\mathbb{R}^{n}\right)} .
$$

Combining these observations, we see that the second embedding holds.

We need the following proposition later, which claims that $\dot{C}^{1, \alpha-1}\left(\mathbb{R}^{n}\right) \hookrightarrow \dot{C}_{\nabla}^{1, \alpha-1}\left(\mathbb{R}^{n}\right)$ for $1<\alpha<2$ in the sense of continuous embedding. 
Proposition 2.3. Let $1<\alpha<2$. Then there exists $C_{\alpha}>0$ such that

$$
\|u\|_{\mathcal{C}_{\nabla}^{1, \alpha-1}\left(\mathbb{R}^{n}\right)} \leq C_{\alpha}\|u\|_{\dot{C}^{1, \alpha-1}\left(\mathbb{R}^{n}\right)} \text { for } u \in \dot{C}^{1, \alpha-1}\left(\mathbb{R}^{n}\right) .
$$

The proof is somehow well known (see [15, Chapter 0] when $n=1$ ). Here for the sake of convenience we include it in the appendix. We will show that this fact is also valid on a domain $\Omega \subset \mathbb{R}^{n}$.

Proposition 2.4. Let $1<\alpha<2$ and $\Omega$ be a domain in $\mathbb{R}^{n}$. Then there exists $C_{\alpha}>0$ such that

$$
\|u\|_{\dot{C}_{\nabla}^{1, \alpha-1}(\Omega)} \leq C_{\alpha}\|u\|_{\dot{C}^{1, \alpha-1}(\Omega)} \quad \text { for } u \in \dot{C}^{1, \alpha-1}(\Omega)
$$

Proof. For any $u \in \dot{C}^{1, \alpha-1}(\Omega)$, there exists an extension $v_{u} \in \dot{C}^{1, \alpha-1}\left(\mathbb{R}^{n}\right)$ of $u$ on $\mathbb{R}^{n}$ such that

$$
\left.v_{u}\right|_{\Omega}=u \quad \text { in } \Phi^{\prime}(\Omega), \quad\|u\|_{\dot{C}^{1, \alpha-1}(\Omega)} \leq\left\|v_{u}\right\|_{\dot{C}^{1, \alpha-1}\left(\mathbb{R}^{n}\right)} \leq 2\|u\|_{\dot{C}^{1, \alpha-1}(\Omega)} .
$$

In particular, $\left.\nabla v_{u}\right|_{\Omega}=\nabla u$ in $\Phi^{\prime}(\Omega)$. By applying Proposition 2.3, we have

$$
\begin{aligned}
& \|u\|_{\dot{C}_{\nabla}^{1, \alpha-1}\left(\Omega ; \mathbb{R}^{n}\right)} \\
& \quad=\inf \left\{\|\nabla v\|_{\dot{C}^{0, \alpha-1}\left(\mathbb{R}^{n} ; \mathbb{R}^{n}\right)} ; v \in \dot{C}_{\nabla}^{1, \alpha-1}\left(\mathbb{R}^{n}\right),\left.(\nabla v)\right|_{\Omega}=\nabla u \text { in } \Phi^{\prime}(\Omega)\right\} \\
& \quad \leq\left\|\nabla v_{u}\right\|_{\dot{C}^{0, \alpha-1}\left(\mathbb{R}^{n} ; \mathbb{R}^{n}\right)}=\left\|v_{u}\right\|_{\dot{C}_{\nabla}^{1, \alpha-1}\left(\mathbb{R}^{n} ; \mathbb{R}^{n}\right)} \\
& \quad \leq C_{\alpha}\left\|v_{u}\right\|_{\dot{C}^{1, \alpha-1}\left(\mathbb{R}^{n}\right)} \leq 2 C_{\alpha}\|u\|_{\dot{C}^{1, \alpha-1}(\Omega)}
\end{aligned}
$$

and obtain the desired result.

Let us establish the following proposition. Here, unlike a bounded domain $\Omega$, for the whole space $\mathbb{R}^{n}$ we adopt the following definition of the norm of $W^{1, n}\left(\mathbb{R}^{n}\right)$ :

$$
\|u\|_{W^{1, n}\left(\mathbb{R}^{n}\right)}=\|u\|_{L^{n}\left(\mathbb{R}^{n}\right)}+\|\nabla u\|_{L^{n}\left(\mathbb{R}^{n}\right)} .
$$

Definition 2.5. One says that a bounded domain $\Omega$ satisfies the strong local Lipschitz condition if $\Omega$ has a locally Lipschitz boundary, that is, each point $x$ on the boundary of $\Omega$ has a neighborhood $U_{x}$ whose intersection with the boundary of $\Omega$ is the graph of a Lipschitz continuous function.

The definition for a general domain is more complicated; see [11] for details.

Proposition 2.6. Let $0<\gamma<\alpha$. Then one has

$$
\|u\|_{B^{\gamma, \infty, \infty}\left(\mathbb{R}^{n}\right)} \leq C_{\gamma}\|u\|_{B^{\alpha, \infty, \infty}\left(\mathbb{R}^{n}\right)}^{\gamma / \alpha}\|u\|_{W^{1, n}\left(\mathbb{R}^{n}\right)}^{1-\gamma / \alpha} \quad \text { for } u \in W^{1, n}\left(\mathbb{R}^{n}\right) \cap B^{\alpha, \infty, \infty}\left(\mathbb{R}^{n}\right)
$$


Furthermore, let $\Omega$ be a bounded domain in $\mathbb{R}^{n}$ satisfying the strong local Lipschitz condition. Then one has

$$
\|u\|_{B^{\gamma, \infty, \infty}(\Omega)} \leq C_{\gamma}\|u\|_{B^{\alpha, \infty}, \infty(\Omega)}^{\gamma / \alpha}\|\nabla u\|_{L^{n}(\Omega)}^{1-\gamma / \alpha} \quad \text { for } u \in W_{0}^{1, n}(\Omega) \cap B^{\alpha, \infty, \infty}(\Omega)
$$

Proposition 2.6 can be obtained directly from a theory of interpolation. However, the proof being simple, we include it for the sake of reader's convenience.

Proof of Proposition 2.6. Let us take $\zeta \in C_{\mathrm{c}}^{\infty}\left(\mathbb{R}^{n}\right)$ so that $\zeta=1$ on $\bar{B}_{4} \backslash B_{1}$ and $\operatorname{supp} \zeta \subset \bar{B}_{8} \backslash B_{1 / 2}$. Set

$$
\zeta_{i}(\xi)=\frac{\xi_{i}}{|\xi|^{2}} \zeta(\xi), \quad \zeta_{i, k}(\xi)=\zeta_{i}\left(\frac{\xi}{2^{k}}\right)=\frac{2^{k} \xi_{i}}{|\xi|^{2}} \zeta\left(\frac{\xi}{2^{k}}\right) \quad \text { for } k \in \mathbb{N} \cup\{0\}, i \in\{1, \ldots, n\}
$$

Recall that $\varphi_{k}^{0}$ is supported on $\bar{B}_{2^{k+2}} \backslash B_{2^{k}}$, and observe that

$$
\varphi_{k}^{0}(\xi)=\frac{1}{2^{k}} \sum_{i=1}^{n} \xi_{i} \zeta_{i, k}(\xi) \varphi_{k}^{0}(\xi)
$$

Hence we have

$$
\begin{aligned}
& \left\|\varphi_{k}^{0}(D) u\right\|_{L^{\infty}\left(\mathbb{R}^{n}\right)} \\
& =\frac{1}{2^{k}}\left\|\sum_{i=1}^{n} \zeta_{i, k}(D) \varphi_{k}^{0}(D) \frac{\partial u}{\partial x_{i}}\right\|_{L^{\infty}\left(\mathbb{R}^{n}\right)} \\
& =\frac{1}{(2 \pi)^{n} 2^{k}}\left\|\sum_{i=1}^{n}\left(\mathcal{F}^{-1} \zeta_{i, k}\right) *\left(\mathcal{F}^{-1} \varphi_{k}^{0}\right) * \frac{\partial u}{\partial x_{i}}\right\|_{L^{\infty}\left(\mathbb{R}^{n}\right)} \\
& \leq \frac{1}{(2 \pi)^{n} 2^{k}}\left\|\mathcal{F}^{-1} \varphi_{k}^{0}\right\|_{L^{1}\left(\mathbb{R}^{n}\right)} \sum_{i=1}^{n}\left\|\mathcal{F}^{-1} \zeta_{i, k}\right\|_{L^{n /(n-1)}\left(\mathbb{R}^{n}\right)}\left\|\frac{\partial u}{\partial x_{i}}\right\|_{L^{n}\left(\mathbb{R}^{n}\right)} \\
& \quad=\frac{1}{(2 \pi)^{n}}\left\|\mathcal{F}^{-1} \varphi^{0}\right\|_{L^{1}\left(\mathbb{R}^{n}\right)} \sum_{i=1}^{n}\left\|\mathcal{F}^{-1} \zeta_{i}\right\|_{L^{n /(n-1)}\left(\mathbb{R}^{n}\right)}\left\|\frac{\partial u}{\partial x_{i}}\right\|_{L^{n}\left(\mathbb{R}^{n}\right)} \\
& \leq C\|\nabla u\|_{L^{n}\left(\mathbb{R}^{n}\right)} \leq C\|u\|_{W^{1, n}\left(\mathbb{R}^{n}\right)} .
\end{aligned}
$$

A similar estimate for $\psi^{0}$ is also available:

$$
\begin{aligned}
\left\|\psi^{0}(D) u\right\|_{L^{\infty}\left(\mathbb{R}^{n}\right)} & =\frac{1}{(2 \pi)^{n / 2}}\left\|\left(\mathcal{F}^{-1} \psi^{0}\right) * u\right\|_{L^{\infty}\left(\mathbb{R}^{n}\right)} \\
& \leq C\|u\|_{L^{n}\left(\mathbb{R}^{n}\right)} \leq C\|u\|_{W^{1, n}\left(\mathbb{R}^{n}\right)} .
\end{aligned}
$$


Hence we have

$$
\begin{aligned}
\|u\|_{B^{\gamma, \infty, \infty}\left(\mathbb{R}^{n}\right)} & =\left\|\psi^{0}(D) u\right\|_{L^{\infty}\left(\mathbb{R}^{n}\right)}+\sup _{k \in \mathbb{N} \cup\{0\}} 2^{\gamma l}\left\|\varphi_{k}^{0}(D) u\right\|_{L^{\infty}\left(\mathbb{R}^{n}\right)} \\
& \leq C_{\gamma} \sup _{k \in \mathbb{N} \cup\{0\}} \min \left\{\frac{1}{2^{(\alpha-\gamma) k}}\|u\|_{B^{\alpha, \infty, \infty}\left(\mathbb{R}^{n)},\right.} 2^{\gamma k}\|u\|_{W^{1, n}\left(\mathbb{R}^{n}\right)}\right\}
\end{aligned}
$$

since $2^{\alpha k}\left\|\varphi_{k}^{0}(D) u\right\|_{L^{\infty}\left(\mathbb{R}^{n}\right)} \leq\|u\|_{B^{\alpha, \infty, \infty}\left(\mathbb{R}^{n}\right)}$. Hence we have

$$
\begin{aligned}
& \|u\|_{B^{r, \infty, \infty}\left(\mathbb{R}^{n}\right)} \leq C_{\gamma} \sup _{t>0} \min \left\{\frac{1}{t^{\alpha-\gamma}}\|u\|_{B^{\alpha, \infty, \infty}\left(\mathbb{R}^{n}\right)}, t t^{\gamma}\|u\|_{W^{1, n}\left(\mathbb{R}^{n}\right)}\right\} \\
& =C_{\gamma}\|u\|_{B^{\alpha, \infty, \infty}\left(\mathbb{R}^{n}\right)}^{\gamma / \alpha}\|u\|_{W^{1, n}\left(\mathbb{R}^{n}\right)}^{1-\gamma / \alpha} \text {. }
\end{aligned}
$$

It remains to prove (2.21). The universal extension theorem obtained by Rychkov [12, Theorem 2.2] yields that there exists a common extension operator $E: W_{0}^{1, n}(\Omega)+B^{r, \infty, \infty}(\Omega) \rightarrow$ $W^{1, n}\left(\mathbb{R}^{n}\right)+B^{r, \infty, \infty}\left(\mathbb{R}^{n}\right)$ such that

$$
\begin{gathered}
\|u\|_{B^{\beta, \infty, \infty}(\Omega)} \leq\|E u\|_{B^{\beta, \infty, \infty}\left(\mathbb{R}^{n}\right)} \leq C_{\beta}\|u\|_{B^{\beta, \infty, \infty}(\Omega)} \quad \text { for } u \in B^{\beta, \infty, \infty}(\Omega), \\
\|\nabla u\|_{L^{n}(\Omega)} \leq\|E u\|_{W^{1, n}\left(\mathbb{R}^{n}\right)} \leq C\|\nabla u\|_{L^{n}(\Omega)} \text { for } u \in W_{0}^{1, n}(\Omega)
\end{gathered}
$$

for all $\gamma \leq \beta<\infty$. Then (2.21) is an immediate consequence of (2.20).

\section{Counterexample for the Inequality}

In this section, we will give the proof of assertion (ii) of Theorems 1.5-1.9. Lemma 2.2 shows that

$$
B^{s, p, \min \{p, q\}}(\Omega) \hookrightarrow F^{s, p, q}(\Omega),
$$

and hence it suffices to consider the case $A^{s, p_{\alpha, s}, q}(\Omega)=B^{s, p_{\alpha, s}, q}(\Omega)$ in view of Proposition 1.2 (i). Furthermore, Lemma 2.2 also shows that

$$
B^{\tilde{s}, p_{\alpha, \tilde{s}}, \min \left\{p_{\alpha, \tilde{s}, q\}}\right.}(\Omega) \hookrightarrow B^{s, p_{\alpha, s}, q}(\Omega) \quad \text { for } \tilde{s}>s,
$$

and hence we have only to consider the case $0<q \leq p_{\alpha, s}=n /(s-\alpha) \leq 1$. Therefore, it suffices to show the following theorem for the proof of (ii) of Theorems 1.5-1.9.

Theorem 3.1. Let $n \geq 2, \alpha>0, s \geq n+\alpha, 0<q \leq p_{\alpha, s}$, and let $\Omega$ be a bounded domain in $\mathbb{R}^{n}$ and $X(\Omega)=B^{s, p_{\alpha, s}, q}(\Omega)$. Assume that either (III) or (IV) holds. Then for any constant $C$, inequality (1.7) fails for some $u \in C_{c}^{\infty}(\Omega)$ with $\|\nabla u\|_{L^{n}(\Omega)}=1$. 
Here and below, we use the notation

$$
\ell(s)=\log (1+s) \quad \text { for } s \geq 0
$$

for short, and then $\ell \circ \ell(s)=\log (1+\log (1+s))$ for $s \geq 0$. We note that inequality (1.7) with $X(\Omega)=B^{s, p_{\alpha, s}, q}(\Omega)$ holds for all $u \in W_{0}^{1, n}(\Omega) \cap B^{s, p_{\alpha, s}, q}(\Omega)$ with $\|\nabla u\|_{L^{n}(\Omega)}=1$ if and only if there exists a constant $C$ independent of $u$ such that $F^{\alpha, s, q}\left[u ; \lambda_{1}, \lambda_{2}\right] \leq C$ holds for all $u \in W_{0}^{1, n}(\Omega) \cap B^{s, p_{\alpha, s}, q}(\Omega) \backslash\{0\}$, where

$$
\begin{array}{r}
F^{\alpha, s, q}\left[u ; \lambda_{1}, \lambda_{2}\right]=\left(\frac{\|u\|_{L^{\infty}(\Omega)}}{\|\nabla u\|_{L^{n}(\Omega)}}\right)^{n /(n-1)}-\lambda_{1} \ell\left(\frac{\|u\|_{B^{s, p_{\alpha, s}, q}(\Omega)}}{\|\nabla u\|_{L^{n}(\Omega)}}\right)-\lambda_{2} \ell \circ \ell\left(\frac{\|u\|_{B^{s, p \alpha_{\alpha}, q, q}(\Omega)}}{\|\nabla u\|_{L^{n}(\Omega)}}\right) \\
\text { for } u \in W_{0}^{1, n}(\Omega) \cap B^{s, p_{\alpha, s}, q}(\Omega) \backslash\{0\} .
\end{array}
$$

For the proof of Theorem 3.1, we have to find a sequence $\left\{u_{j}\right\}_{j=1}^{\infty} \subset C_{\mathrm{c}}^{\infty}(\Omega) \backslash\{0\}$ such that $F^{\alpha, s, q}\left[u_{j} ; \lambda_{1}, \lambda_{2}\right] \rightarrow \infty$ as $j \rightarrow \infty$ under assumption (III) or (IV). In the case that $\Omega=\mathbb{R}^{n}$ and that all the functions are supported in $B_{1}$, we can choose such a sequence.

Lemma 3.2. Let $n \geq 2, \alpha>0, s \geq n+\alpha, 0<q \leq p_{\alpha, s}$, and $\Omega=\mathbb{R}^{n}$. Then there exists a family of functions $\left\{u_{j}\right\}_{j=1}^{\infty} \subset C_{c}^{\infty}\left(\mathbb{R}^{n}\right) \backslash\{0\}$ with supp $u_{j} \subset B_{1}$ for all $j \in \mathbb{N}$ such that

$$
F^{\alpha, s, q}\left[u_{j} ; \lambda_{1}, \lambda_{2}\right] \longrightarrow \infty \text { as } j \longrightarrow \infty
$$

under assumption (III) or (IV) of Theorem 3.1.

We can now prove Theorem 3.1 once we accept Lemma 3.2.

Proof of Theorem 3.1. Examining (1.7) fails, so we may assume that $\lambda_{1}, \lambda_{2} \geq 0$. Fix $z_{0} \in \Omega$ and $R_{0} \geq 1$ such that

$$
B=\left\{x \in \mathbb{R}^{n} ;\left|x-z_{0}\right|<\frac{1}{R_{0}}\right\} \subset \Omega .
$$

Let $\left\{u_{j}\right\}_{j=1}^{\infty}$ be a family of functions as in Lemma 3.2. If we set

$$
v_{j}(x)= \begin{cases}u_{j}\left(R_{0}\left(x-z_{0}\right)\right) & \text { for } x \in B, \\ 0 & \text { for } x \in \Omega \backslash B,\end{cases}
$$

then $v_{j} \in C_{\mathrm{c}}^{\infty}(\Omega)$, and there exists a constant $C_{\alpha, s, R_{0}} \geq 1$ such that

$$
\begin{gathered}
\left\|v_{j}\right\|_{L^{\infty}(\Omega)}=\left\|u_{j}\right\|_{L^{\infty}\left(\mathbb{R}^{n}\right)^{\prime}} \quad\left\|\nabla v_{j}\right\|_{L^{n}(\Omega)}=\left\|\nabla u_{j}\right\|_{L^{n}\left(\mathbb{R}^{n}\right)^{\prime}} \\
\left\|v_{j}\right\|_{B^{s, p, \alpha, s, q}(\Omega)} \leq C_{\alpha, s, R_{0}}\left\|u_{j}\right\|_{B^{s, p \alpha_{\alpha}, s, q\left(\mathbb{R}^{n}\right)^{n}} .}
\end{gathered}
$$


The first and the second equalities are immediate, while the third inequality is a direct consequence of the fact that the dilation $u \mapsto u\left(R_{0} \cdot\right)$ is an isomorphism over $B^{s, p_{\alpha, s}, q}\left(\mathbb{R}^{n}\right)$. Using (1.8) and the fact that $\lambda_{1}, \lambda_{2} \geq 0$, we have

$$
\begin{aligned}
F^{\alpha, s, q}\left[u_{j} ; \lambda_{1}, \lambda_{2}\right] \leq & \left(\frac{\left\|u_{j}\right\|_{L^{\infty}\left(\mathbb{R}^{n}\right)}}{\left\|\nabla u_{j}\right\|_{L^{n}\left(\mathbb{R}^{n}\right)}}\right)^{n /(n-1)}-\lambda_{1} \ell\left(\frac{1}{C_{\alpha, s, R_{0}}} \frac{\left\|u_{j}\right\|_{B^{s, p, \alpha, s, q}\left(\mathbb{R}^{n}\right)}}{\left\|\nabla u_{j}\right\|_{L^{n}\left(\mathbb{R}^{n}\right)}}\right) \\
& -\lambda_{2} \ell \circ \ell\left(\frac{1}{C_{\alpha, s, R_{0}}} \frac{\left\|u_{j}\right\|_{B^{s, p \alpha, s, q}\left(\mathbb{R}^{n}\right)}}{\left\|\nabla u_{j}\right\|_{L^{n}\left(\mathbb{R}^{n}\right)}}\right) \\
\leq & \left(\frac{\left\|v_{j}\right\|_{L^{\infty}(\Omega)}}{\left\|\nabla v_{j}\right\|_{L^{n}(\Omega)}}\right)^{n /(n-1)}-\lambda_{1} \ell\left(\frac{\left\|v_{j}\right\|_{B^{s, p}, s, q, q}(\Omega)}{\left\|\nabla v_{j}\right\|_{L^{n}(\Omega)}}\right) \\
& -\lambda_{2} \ell \circ \ell\left(\frac{\left\|v_{j}\right\|_{B^{s, p \alpha, s, q}(\Omega)}}{\left\|\nabla v_{j}\right\|_{L^{n}(\Omega)}}\right)+C_{\alpha, s, R_{0}, \lambda_{1}, \lambda_{2}} \\
= & F^{\alpha, s, q}\left[v_{j} ; \lambda_{1}, \lambda_{2}\right]+C_{\alpha, s, R_{0}, \lambda_{1}, \lambda_{2},}
\end{aligned}
$$

from which we conclude that $F^{\alpha, s, q}\left[v_{j} ; \lambda_{1}, \lambda_{2}\right] \rightarrow \infty$ as $j \rightarrow \infty$.

We now concentrate on the proof of Lemma 3.2, and we first prepare several lemmas.

Let $\widetilde{\varphi}^{0} \in C_{\mathrm{c}}^{\infty}((0, \infty))$ be a smooth function that is nonnegative, supported on the interval $[1,4]$ and satisfies

$$
\sum_{l=-\infty}^{\infty} \tilde{\varphi}^{0}\left(2^{l+2} t\right)=1 \quad \text { for } t>0
$$

Observe that (3.10) forces $\tilde{\varphi}^{0}(2)=1$.

Proposition 3.3. (i) It holds

$$
\chi_{\left[1 / 2^{j+1}, 1 / 4\right]}(t) \leq \sum_{l=1}^{j} \widetilde{\varphi}^{0}\left(2^{l+2} t\right) \leq \chi_{\left[1 / 2^{j+2}, 1 / 2\right]}(t) \quad \text { for } j \in \mathbb{N} .
$$

(ii) It holds

$$
\int_{0}^{\infty} \frac{\tilde{\varphi}^{0}(t)}{t} d t=\log 2
$$

Proof. (i) In view of the size of the support of $\widetilde{\varphi}^{0}$, we easily obtain (3.11).

(ii) If we integrate both the sides of inequality (3.11), then we have

$$
\frac{1}{j} \int_{1 / 2^{j+1}}^{1 / 4} \frac{1}{t} d t \leq \frac{1}{j} \sum_{l=1}^{j} \int_{0}^{\infty} \frac{\tilde{\varphi}^{0}\left(2^{l+2} t\right)}{t} d t=\int_{0}^{\infty} \frac{\tilde{\varphi}^{0}(t)}{t} d t \leq \frac{1}{j} \int_{1 / 2^{j+2}}^{1 / 2} \frac{1}{t} d t .
$$


As a consequence, it follows that

$$
(\log 2)\left(1-\frac{1}{j}\right) \leq \int_{0}^{\infty} \frac{\tilde{\varphi}^{0}(t)}{t} d t \leq(\log 2)\left(1+\frac{1}{j}\right)
$$

A passage to the limit as $j \rightarrow \infty$ therefore yields (3.12).

Define

$$
w_{l}(x)=\int_{|x|}^{\infty} \frac{\tilde{\varphi}^{0}\left(2^{l+2} t\right)}{t} d t \quad \text { for } x \in \mathbb{R}^{n}, l \in \mathbb{N} .
$$

Note that $w_{l}=w_{1}\left(2^{l-1} \cdot\right)$. Set

$$
u_{j}(x)=\frac{1}{(\log 2) j} \sum_{l=1}^{j} w_{l}(x) \quad \text { for } x \in \mathbb{R}^{n}, j \in \mathbb{N} .
$$

We also note that supp $u_{j} \subset \bar{B}_{1 / 2}$ since supp $w_{l} \subset \bar{B}_{1 / 2^{l}}$.

When we are going to specify the best constant,(3.19) is the heart of the matter.

Lemma 3.4. Let $n \geq 2$ and $0<p<\infty$. Then one has

$$
\begin{gathered}
\left\|u_{j}\right\|_{L^{\infty}\left(\mathbb{R}^{n}\right)}=1 \quad \text { for } j \in \mathbb{N}, \\
\frac{(1-1 / j)^{p}}{n \Lambda_{1}^{n-1} 2^{n(j+1)}} \leq\left\|u_{j}\right\|_{L^{p}\left(\mathbb{R}^{n}\right)}^{p} \leq \frac{(1+1 / j)^{p}}{n \Lambda_{1}^{n-1}} \text { for } j \in \mathbb{N}, \\
\frac{1-1 / j}{\left((\log 2) \Lambda_{1}\right)^{n-1} j^{n-1}} \leq\left\|\nabla u_{j}\right\|_{L^{n}\left(\mathbb{R}^{n}\right)}^{n} \leq \frac{1+1 / j}{\left((\log 2) \Lambda_{1}\right)^{n-1} j^{n-1}} \quad \text { for } j \in \mathbb{N} .
\end{gathered}
$$

Proof. It is not so hard to prove (3.17). Indeed, a change of variables yields

$$
\left\|u_{j}\right\|_{L^{\infty}\left(\mathbb{R}^{n}\right)}=u_{j}(0)=\frac{1}{(\log 2) j} \int_{0}^{\infty} \sum_{l=1}^{j} \frac{\tilde{\varphi}^{0}\left(2^{l+2} t\right)}{t} d t=\frac{1}{\log 2} \int_{0}^{\infty} \frac{\tilde{\varphi}^{0}(t)}{t} d t
$$

Thus, we obtain (3.17) by applying (3.12).

We next verify (3.18). Recall that $\Lambda_{1}$ is defined by $\Lambda_{1}=1 / \omega_{n-1}^{1 /(n-1)}$. If we insert the definitions (3.15) and (3.16), then we have

$$
\left\|u_{j}\right\|_{L^{p}\left(\mathbb{R}^{n}\right)}^{p}=\frac{1}{\Lambda_{1}^{n-1}((\log 2) j)^{p}} \int_{0}^{1}\left(\int_{r}^{\infty} \frac{1}{t} \sum_{l=1}^{j} \tilde{\varphi}^{0}\left(2^{l+2} t\right) d t\right)^{p} r^{n-1} d r .
$$


Using (3.11), we have

$$
\begin{aligned}
\left\|u_{j}\right\|_{L^{p}\left(\mathbb{R}^{n}\right)}^{p} & \leq \frac{1}{\Lambda_{1}^{n-1}((\log 2) j)^{p}} \int_{0}^{1}\left(\int_{r}^{\infty} \frac{X_{\left[1 / 2^{j+2}, 1 / 2\right]}(t)}{t} d t\right)^{p} r^{n-1} d r \\
& \leq \frac{1}{\Lambda_{1}^{n-1}((\log 2) j)^{p}} \int_{0}^{1}\left(\int_{1 / 2^{j+2}}^{1 / 2} \frac{1}{t} d t\right)^{p} r^{n-1} d r \\
& =\frac{(1+1 / j)^{p}}{n \Lambda_{1}^{n-1}}, \\
\left\|u_{j}\right\|_{L^{p}\left(\mathbb{R}^{n}\right)}^{p} & \geq \frac{1}{\Lambda_{1}^{n-1}((\log 2) j)^{p}} \int_{0}^{1}\left(\int_{r}^{\infty} \frac{X_{\left[1 / 2^{j+1}, 1 / 4\right]}(t)}{t} d t\right)^{p} r^{n-1} d r \\
& \geq \frac{1}{\Lambda_{1}^{n-1}((\log 2) j)^{p}} \int_{0}^{1 / 2^{j+1}}\left(\int_{1 / 2^{j+1}}^{1 / 4} \frac{1}{t} d t\right)^{p} r^{n-1} d r \\
& =\frac{(1-1 / j)^{p}}{n \Lambda_{1}^{n-1} 2^{n(j+1)}} .
\end{aligned}
$$

Equation (3.19) is a simple but delicate inequality, since we need to take a full advantage of the definition of (1.3) and the equality

$$
\nabla u_{j}(x)=-\frac{1}{(\log 2) j}\left(\sum_{l=1}^{j} \tilde{\varphi}^{0}\left(2^{l+2}|x|\right)\right) \frac{x}{|x|^{2}} .
$$

Also, a direct calculation shows that

$$
\left\|\nabla u_{j}\right\|_{L^{n}\left(\mathbb{R}^{n}\right)}^{n}=\frac{1}{\Lambda_{1}^{n-1}((\log 2) j)^{n}} \int_{0}^{1}\left(\sum_{l=1}^{j} \widetilde{\varphi}^{0}\left(2^{l+2} t\right)\right)^{n} \frac{1}{t} d t
$$

By using (3.11), we have

$$
\begin{aligned}
& \left\|\nabla u_{j}\right\|_{L^{n}\left(\mathbb{R}^{n}\right)}^{n} \leq \frac{1}{\Lambda_{1}^{n-1}((\log 2) j)^{n}} \int_{1 / 2^{j+2}}^{1 / 2} \frac{1}{t} d t=\frac{1+1 / j}{\left((\log 2) \Lambda_{1}\right)^{n-1} j^{n-1}}, \\
& \left\|\nabla u_{j}\right\|_{L^{n}\left(\mathbb{R}^{n}\right)}^{n} \geq \frac{1}{\Lambda_{1}^{n-1}((\log 2) j)^{n}} \int_{1 / 2^{j+1}}^{1 / 4} \frac{1}{t} d t=\frac{1-1 / j}{\left((\log 2) \Lambda_{1}\right)^{n-1} j^{n-1}} .
\end{aligned}
$$

Let us estimate the Besov norm of $u_{j}$, which is the most delicate in this proof. 
Lemma 3.5. Let $n \geq 2, \alpha>0, s \geq n+\alpha$, and $0<q \leq p_{\alpha, s}$. Then one has

$$
\left\|u_{j}\right\|_{B^{s, p \alpha, s, q\left(\mathbb{R}^{n}\right)}} \leq C_{\alpha, s, q} \frac{2^{\alpha j}}{j} \quad \text { for } j \in \mathbb{N} .
$$

Lemma 3.5 is reduced to Propositions 3.6 and 3.7 below, which concern lower frequency part and higher frequency part, respectively.

Proposition 3.6. Take $\psi^{0}$ satisfying (1.12). Let $n \geq 2, \alpha>0$, and $s \geq n+\alpha$. Then there exists a constant $C_{\alpha, s}$ such that

$$
\left\|\psi^{0}(D) u_{j}\right\|_{L^{p_{\alpha, s}\left(\mathbb{R}^{n}\right)}} \leq C_{\alpha, s} \quad \text { for } j \in \mathbb{N} .
$$

Proposition 3.7. Take $\varphi^{0}$ satisfying (1.12). Let $n \geq 2, \alpha>0, s \geq n+\alpha$, and $0<q \leq p_{\alpha, s}$. Then there exists a constant $C_{\alpha, s, q}$ such that

$$
\left(\sum_{k=0}^{\infty} 2^{s q k}\left\|\varphi_{k}^{0}(D) u_{j}\right\|_{L^{p_{\alpha, s}\left(\mathbb{R}^{n}\right)}}^{q}\right)^{1 / q} \leq C_{\alpha, s, q} \frac{2^{\alpha j}}{j} \quad \text { for } j \in \mathbb{N} .
$$

For $m>0$, let us set

$$
\phi^{m}(x)=\frac{1}{(1+|x|)^{n+m}} \quad \text { for } x \in \mathbb{R}^{n}
$$

and estimate $\left\|\phi^{m} * \chi_{B_{R}}\right\|_{L^{p}\left(\mathbb{R}^{n}\right)}$ crudely, where $0<p \leq \infty$ and $R>0$. Let $s_{+}$denote the positive part of $s \in \mathbb{R}$, that is, $s_{+}=\max \{s, 0\}$.

Proposition 3.8. If $m>n(1 / p-1)_{+}, 0<p \leq \infty$, and $R>0$, then there exists a constant $C_{p, m}>0$ such that

$$
\left\|\phi^{m} * X_{B_{R}}\right\|_{L^{p}\left(\mathbb{R}^{n}\right)} \leq C_{p, m} \max \left\{R^{n}, R^{n / p}\right\} .
$$

Proof. Let us decompose the estimate of $\left\|\phi^{m} * X_{B_{R}}\right\|_{L^{p}\left(\mathbb{R}^{n}\right)}$ according to $B_{2 R}$. As for the estimate inside $B_{2 R}$, we have

$$
\begin{aligned}
\left\|\phi^{m} * X_{B_{R}}\right\|_{L^{p}\left(B_{2 R}\right)} & =\left(\int_{B_{2 R}}\left(\int_{B_{R}} \frac{1}{(1+|x-z|)^{n+m}} d z\right)^{p} d x\right)^{1 / p} \\
& \leq\left(\int_{B_{2 R}}\left(\int_{\mathbb{R}^{n}} \frac{1}{(1+|x-z|)^{n+m}} d z\right)^{p} d x\right)^{1 / p} \\
& =\left(\frac{2 \omega_{n-1}}{n}\right)^{n / p}\left\|\phi^{m}\right\|_{L^{1}\left(\mathbb{R}^{n}\right)} R^{n / p}
\end{aligned}
$$


Let us turn to the estimate outside $B_{2 R}$. Since

$$
\begin{array}{r}
|x| \leq|x-z|+|z|<|x-z|+R \leq|x-z|+|x|-|z| \leq 2|x-z| \\
\text { for } x \in \mathbb{R}^{n} \backslash B_{2 R}, \quad z \in B_{R},
\end{array}
$$

we have

$$
\begin{aligned}
\left\|\phi^{m} * X_{B_{R}}\right\|_{L^{p}\left(\mathbb{R}^{n} \backslash B_{2 R}\right)} & =\left(\int_{\mathbb{R}^{n} \backslash B_{2 R}}\left(\int_{B_{R}} \frac{1}{(1+|x-z|)^{n+m}} d z\right)^{p} d x\right)^{1 / p} \\
& \leq\left(\int_{\mathbb{R}^{n}}\left(\int_{B_{R}} \frac{1}{(1+|x| / 2)^{n+m}} d z\right)^{p} d x\right)^{1 / p} \\
& =\left(\frac{2^{1 / p} \omega_{n-1}}{n}\right)^{n}\left\|\phi^{m}\right\|_{L^{p}\left(\mathbb{R}^{n}\right)} R^{n} .
\end{aligned}
$$

Thus we have proved the assertion.

We first prove Proposition 3.6. We abbreviate $\chi_{B_{2 l}}=\chi_{l}$ for $l \in \mathbb{Z}$.

Proof of Proposition 3.6. Choose $m_{\alpha, s} \in \mathbb{N}$ satisfying $m_{\alpha, s}>n\left(1 / p_{\alpha, s}-1\right)=s-\alpha-n$. Since $\mathcal{F}^{-1} \psi^{0} \in \mathcal{S}\left(\mathbb{R}^{n}\right)$, we have

$$
\left|\mathcal{F}^{-1} \psi^{0}(x)\right| \leq C_{\alpha, s} \phi^{m_{\alpha, s}}(x) \text { for } x \in \mathbb{R}^{n}
$$

It follows from (3.17) that $u_{j}(x) \leq \chi_{0}(x)$ for $x \in \mathbb{R}^{n}$. Applying Proposition 3.8, we have

$$
\begin{aligned}
\left\|\psi^{0}(D) u_{j}\right\|_{L^{p_{\alpha, s}\left(\mathbb{R}^{n}\right)}} & =\frac{1}{(2 \pi)^{n / 2}}\left\|\left(\mathcal{F}^{-1} \psi^{0}\right) * u_{j}\right\|_{L^{p_{\alpha, s}\left(\mathbb{R}^{n}\right)}} \\
& \leq C_{\alpha, s}\left\|\phi^{m_{\alpha, s}} * X_{0}\right\|_{L^{p_{\alpha, s}\left(\mathbb{R}^{n}\right)}} \leq C_{\alpha, s} .
\end{aligned}
$$

Let us turn to proving Proposition 3.7.

Proof of Proposition 3.7. Since $\varphi_{k}$ does not contain the origin as its support, we can define smooth functions $\varphi^{N}(\xi), \varphi_{k}^{N}(\xi) \in C_{\mathrm{c}}^{\infty}\left(\mathbb{R}^{n}\right)$ by

$$
\begin{array}{r}
\varphi^{N}(\xi)=\frac{\varphi^{0}(\xi)}{|\xi|^{2 N}}, \quad \varphi_{k}^{N}(\xi)=\varphi^{N}\left(\frac{\xi}{2^{k}}\right)=\left(\frac{2^{k}}{|\xi|}\right)^{2 N} \varphi^{0}\left(\frac{\xi}{2^{k}}\right) \\
\text { for } \xi \in \mathbb{R}^{n}, N \in \mathbb{N}, k \in \mathbb{N} \cup\{0\} .
\end{array}
$$


A direct calculation shows that

$$
2^{2 N k} \varphi_{k}^{0}(D) w_{l}(x)=\varphi_{k}^{N}(D)(-\Delta)^{N} w_{l}(x),
$$

where $\Delta$ denotes the Laplacian on $\mathbb{R}^{n}$. Keeping (3.37) in mind, let us calculate $\left\|\varphi_{k}^{N}(D)(-\Delta)^{N} w_{l}\right\|_{L^{p_{\alpha, s}\left(\mathbb{R}^{n}\right)}}$. Note that the self-similarity $w_{l}=w_{1}\left(2^{l-1} \cdot\right)$ yields

$$
\left|(-\Delta)^{N} w_{l}(x)\right|=2^{2 N(l-1)}\left|\left[(-\Delta)^{N} w_{1}\right]\left(2^{l-1} x\right)\right| \leq C_{N} 2^{2 N l} X_{-l}(x) \text { for } x \in \mathbb{R}^{n} .
$$

Choose $m_{\alpha, s} \in \mathbb{N}$ satisfying $m_{\alpha, s}>n\left(1 / p_{\alpha, s}-1\right)=s-\alpha-n$. Since $\mathcal{F}^{-1} \varphi^{N} \in \mathcal{S}\left(\mathbb{R}^{n}\right)$, we have

$$
\left|\mathcal{F}^{-1} \varphi^{N}(x)\right| \leq C_{\alpha, s, N} \phi^{m_{\alpha, s}}(x) \text { for } x \in \mathbb{R}^{n},
$$

and hence

$$
\left|\mathcal{F}^{-1} \varphi_{k}^{N}(x)\right|=2^{n k}\left|\left[\mathcal{F}^{-1} \varphi^{N}\right]\left(2^{k} x\right)\right| \leq C_{\alpha, s, N} 2^{n k} \phi^{m_{\alpha, s}}\left(2^{k} x\right) \quad \text { for } x \in \mathbb{R}^{n} .
$$

As a result, we obtain

$$
\begin{aligned}
\left|\varphi_{k}^{N}(D)(-\Delta)^{N} w_{l}(x)\right| & =\frac{1}{(2 \pi)^{n / 2}}\left|\left(\mathcal{F}^{-1} \varphi_{k}^{N}\right) *(-\Delta)^{N} w_{l}(x)\right| \\
& \leq C_{\alpha, s, N} 2^{2 N l+n k}\left|\left[\phi^{m_{\alpha, s}}\left(2^{k} \cdot\right)\right] * X_{-l}(x)\right| \\
& =C_{\alpha, s, N} 2^{2 N l+n k}\left|\left[\phi^{m_{\alpha, s}}\left(2^{k} \cdot\right)\right] *\left[\chi_{k-l}\left(2^{k} \cdot\right)\right](x)\right| \\
& =C_{\alpha, s, N} 2^{2 N l}\left|\left[\phi^{m_{\alpha, s}} * x_{k-l}\right]\left(2^{k} x\right)\right| \text { for } x \in \mathbb{R}^{n}
\end{aligned}
$$

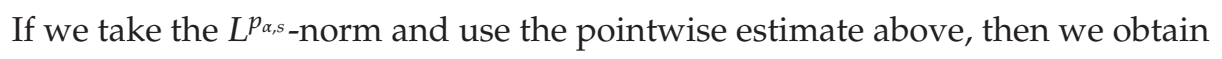

$$
\begin{aligned}
2^{s k}\left\|\varphi_{k}^{0}(D) w_{l}\right\|_{L^{p_{\alpha, s}\left(\mathbb{R}^{n}\right)}} & =2^{(s-2 N) k}\left\|\varphi_{k}^{N}(D)(-\Delta)^{N} w_{l}\right\|_{L^{p_{\alpha, s}\left(\mathbb{R}^{n}\right)}} \\
& \leq C_{\alpha, s, N} 2^{2 N(l-k)+s k}\left\|\left(\phi^{m_{\alpha, s}} * X_{k-l}\right)\left(2^{k} \cdot\right)\right\|_{L^{p_{\alpha, s}\left(\mathbb{R}^{n}\right)}} \\
& =C_{\alpha, s, N} 2^{2 N(l-k)+\alpha k}\left\|\phi^{m_{\alpha, s}} * \chi_{k-l}\right\|_{L^{p_{\alpha, s}\left(\mathbb{R}^{n}\right)}} .
\end{aligned}
$$

Since $m_{\alpha, s}>n\left(1 / p_{\alpha, s}-1\right)$ and $\alpha+n / p_{\alpha, s}=s$, by Proposition 3.8 we have

$$
\begin{array}{cc}
2^{s k}\left\|\varphi_{k}^{0}(D) w_{l}\right\|_{L^{p_{\alpha, s}\left(\mathbb{R}^{n}\right)}} \leq C_{\alpha, s, N} 2^{\alpha l-(\alpha+n-2 N)(l-k)} & \text { for } l \geq k, \\
2^{s k}\left\|\varphi_{k}^{0}(D) w_{l}\right\|_{L^{p_{\alpha, s}\left(\mathbb{R}^{n}\right)}} \leq C_{\alpha, s, N} 2^{\alpha l-(2 N-s)(k-l)} & \text { for } l \leq k
\end{array}
$$


for all $N \in \mathbb{N}$. By letting $N=1$ in (3.43) and $N=\lfloor(s+\alpha) / 2\rfloor+1$ in (3.44), we obtain

$$
2^{s k}\left\|\varphi_{k}^{0}(D) w_{l}\right\|_{L^{p_{\alpha, s}\left(\mathbb{R}^{n}\right)}} \leq C_{\alpha, s} 2^{\alpha(l-|l-k|)} \quad \text { for } l \in \mathbb{N}
$$

because $\min \{\alpha+n-2,2\lfloor(s+\alpha) / 2\rfloor+2-s\} \geq \alpha$, where $[(s+\alpha) / 2\rfloor$ denotes the integer part of $(s+\alpha) / 2$. Since $u_{j}=\sum_{l=1}^{j} w_{l}$, we have

$$
\left(\sum_{k=0}^{\infty} 2^{s q k}\left\|\varphi_{k}^{0}(D) u_{j}\right\|_{L^{p \alpha, s}\left(\mathbb{R}^{n}\right)}^{q}\right)^{1 / q} \leq \frac{1}{(\log 2) j}\left(\sum_{k=0}^{\infty} \sum_{l=1}^{j} 2^{s q k}\left\|\varphi_{k}^{0}(D) w_{l}\right\|_{L^{p \alpha, s}\left(\mathbb{R}^{n}\right)}^{q}\right)^{1 / q} .
$$

Thus by using (3.45) and $q \leq p_{\alpha, s} \leq 1$, we have

$$
\begin{aligned}
& \left(\sum_{k=0}^{\infty} 2^{s q k}\left\|\varphi_{k}^{0}(D) u_{j}\right\|_{L^{p \alpha, s}\left(\mathbb{R}^{n}\right)}^{q}\right)^{1 / q} \\
& \leq \frac{C_{\alpha, s}}{j}\left(\sum_{k=0}^{\infty} \sum_{l=1}^{j} 2^{\alpha q(l-|l-k|)}\right)^{1 / q}=\frac{C_{\alpha, s}}{j}\left(\sum_{l=1}^{j} \sum_{k=0}^{\infty} 2^{\alpha q(l-|k-l|)}\right)^{1 / q} \\
& \quad=\frac{C_{\alpha, s}}{j}\left(\sum_{l=1}^{j} 2^{\alpha q l} \frac{2^{\alpha q}+1-1 / 2^{\alpha(l-1) q}}{2^{\alpha q}-1}\right)^{1 / q} \\
& \leq \frac{C_{\alpha, s, q}}{j}\left(\sum_{l=1}^{j} 2^{\alpha q l}\right)^{1 / q} \leq C_{\alpha, s, q} \frac{2^{\alpha j}}{j} .
\end{aligned}
$$

Thus we obtain the desired conclusion.

Finally we prove Lemma 3.2.

Proof of Lemma 3.2. Let $j_{\alpha} \geq e^{n}+1$ be sufficiently large so that $2^{\alpha j_{\alpha}} \geq\left(j_{\alpha}-1\right)^{1 / n}$. We estimate $F^{\alpha, s, q}\left[u_{j} ; \lambda_{1}, \lambda_{2}\right]$ from below for $j \geq j_{\alpha}$. We have from (3.17) and (3.19) that

$$
\left(\frac{\left\|u_{j}\right\|_{L^{\infty}\left(\mathbb{R}^{n}\right)}}{\left\|\nabla u_{j}\right\|_{L^{n}\left(\mathbb{R}^{n}\right)}}\right)^{n /(n-1)} \geq \frac{(\log 2) \Lambda_{1} j}{(1+1 / j)^{1 /(n-1)}}
$$

It was an elementary arithmetic to deduce (1.8). Another elementary arithmetic we need is

$$
\begin{gathered}
\ell(t) \leq \log t+\log 2 \leq t \quad \text { for } t \geq 1 \\
\ell(r+t) \leq \log r+\log t+\log 3 \text { for } r, t \geq 1
\end{gathered}
$$


Boundary Value Problems

We deduce from Lemma 3.5 that

$$
\ell\left(\frac{\left\|u_{j}\right\|_{B^{s, p \alpha, s, q}\left(\mathbb{R}^{n}\right)}}{\left\|\nabla u_{j}\right\|_{L^{n}\left(\mathbb{R}^{n}\right)}}\right) \leq \ell\left(C_{\alpha, s, q} \frac{2^{\alpha j}}{(j-1)^{1 / n}}\right)
$$

We estimate the right-hand side to obtain The result is

$$
\begin{gathered}
\ell\left(\frac{\left\|u_{j}\right\|_{B^{s, p, s, s, q\left(\mathbb{R}^{n}\right)}}}{\left\|\nabla u_{j}\right\|_{L^{n}\left(\mathbb{R}^{n}\right)}}\right) \\
\leq \ell\left(\frac{2^{\alpha j}}{(j-1)^{1 / n}}\right)+C_{\alpha, s, q} \leq \log \left(\frac{2^{\alpha j}}{(j-1)^{1 / n}}\right)+C_{\alpha, s, q} \\
\quad=(\log 2) \alpha j-\frac{1}{n} \log (j-1)+C_{\alpha, s, q} \leq(\log 2) \alpha j-\frac{1}{n} \log j+C_{\alpha, s, q} \\
\ell \circ \ell\left(\frac{\left\|u_{j}\right\|_{B^{s, p \alpha, s, q\left(\mathbb{R}^{n}\right)}}}{\left\|\nabla u_{j}\right\|_{L^{n}\left(\mathbb{R}^{n}\right)}}\right) \leq \ell\left((\log 2) \alpha j-\frac{1}{n} \log j+C_{\alpha, s, q}\right) .
\end{gathered}
$$

We may and do remove $-(1 / n) \log j$ to obtain the conclusion as follows:

$$
\begin{aligned}
& \ell \circ \ell\left(\frac{\left\|u_{j}\right\|_{B^{s, p \alpha, s, q}\left(\mathbb{R}^{n}\right)}}{\left\|\nabla u_{j}\right\|_{L^{n}\left(\mathbb{R}^{n}\right)}}\right) \\
& \quad \leq \ell\left((\log 2) \alpha j+C_{\alpha, s, q}\right) \leq \log ((\log 2) \alpha j)+C_{\alpha, s, q}=\log j+C_{\alpha, s, q} .
\end{aligned}
$$

We next invoke the fact that

$$
\frac{h}{1-1 /(1+h)^{1 /(n-1)}}=\sum_{k=1}^{n-1}(1+h)^{k /(n-1)}>n-1 \quad \text { for } h>0
$$

By putting $h=1 / j$, we have

$$
\frac{1}{(1+1 / j)^{1 /(n-1)}}>1-\frac{1}{(n-1) j} \quad \text { for } j \geq 1
$$


Therefore, we obtain

$$
\begin{aligned}
& F^{\alpha, s, q}\left[u_{j} ; \lambda_{1}, \lambda_{2}\right] \\
& \quad \geq(\log 2) \Lambda_{1}\left(\frac{1}{(1+1 / j)^{1 /(n-1)}}-\frac{\alpha \lambda_{1}}{\Lambda_{1}}\right) j+\left(\frac{\lambda_{1}}{n}-\lambda_{2}\right) \log j-C_{\alpha, s, q, \lambda_{1}, \lambda_{2}} \\
& >(\log 2) \Lambda_{1}\left(\left(1-\frac{\alpha \lambda_{1}}{\Lambda_{1}}\right) j-\frac{1}{n-1}\right)+\left(\frac{\lambda_{1}}{n}-\lambda_{2}\right) \log j-C_{\alpha, s, q, \lambda_{1}, \lambda_{2}} \\
& \quad \longrightarrow \infty \text { as } j \longrightarrow \infty
\end{aligned}
$$

under assumption (III) or (IV).

Remark 3.9. As we stated in Remark 1.12, if $r>n /(n-1)$, then for any $\lambda_{1}>0$ and any constant $C$, (1.19) does not hold for some $u \in W_{0}^{1, n}(\Omega) \cap X(\Omega)$ with $\|\nabla u\|_{L^{n}(\Omega)}=1$. To see this, let $r=(1+\varepsilon) n /(n-1), \varepsilon>0$, and define

$$
\begin{array}{r}
F^{\alpha, s, q, \varepsilon}\left[u ; \lambda_{1}\right]=\left(\frac{\|u\|_{L^{\infty}(\Omega)}}{\|\nabla u\|_{L^{n}(\Omega)}}\right)^{(1+\varepsilon) n /(n-1)}-\lambda_{1} \ell\left(\frac{\|u\|_{B^{s, p \alpha_{\alpha}, s, q}(\Omega)}}{\|\nabla u\|_{L^{n}(\Omega)}}\right) \\
\text { for } u \in W_{0}^{1, n}(\Omega) \cap B_{0}^{s, p_{\alpha, s}, q}(\Omega) \backslash\{0\}
\end{array}
$$

instead of $F^{\alpha, s, q}\left[u ; \lambda_{1}, \lambda_{2}\right]$. We argue as in the proof of Lemma 3.2 to obtain

$$
\begin{aligned}
F^{\alpha, s, q, \varepsilon}\left[u_{j} ; \lambda_{1}\right] & \geq\left(\frac{(\log 2) \Lambda_{1} j}{(1+1 / j)^{1 /(n-1)}}\right)^{1+\varepsilon}-(\log 2) \alpha \lambda_{1} j+\frac{\lambda_{1}}{n} \log j-\lambda_{1} C_{\alpha, s, q} \\
& \geq\left(\frac{(\log 2) \Lambda_{1} j}{2}\right)^{1+\varepsilon}-(\log 2) \alpha \lambda_{1} j+\frac{\lambda_{1}}{n} \log j-\lambda_{1} C_{\alpha, s, q} \\
& \longrightarrow \infty \text { as } j \longrightarrow \infty,
\end{aligned}
$$

which provides the assertion above.

\section{Establishment of the Inequality (I)}

In this section, we will prove Theorem 1.5(i) and Theorem 1.7(i).

The following theorem, which provides the corresponding result for $X(\Omega)=C^{0, \alpha}(\Omega)$, is essential for proving them.

Theorem 4.1 ([8, Theorem 1.1]). Let $n \geq 2,0<\alpha \leq 1$, let $\Omega$ be a bounded domain in $\mathbb{R}^{n}$, and $X(\Omega)=\dot{C}^{0, \alpha}(\Omega)$. Assume that either (I) or (II) holds. Then there exists a constant $C$ such that the inequality (1.7) holds for all $u \in W_{0}^{1, n}(\Omega) \cap \dot{C}^{0, \alpha}(\Omega)$ with $\|\nabla u\|_{L^{n}(\Omega)}=1$.

We should mention that Ibrahim et al. [16, Theorems 1.3 and 1.4] have already obtained a similar result in the 2-dimensional case. 
First, we prove Theorem 1.5(i). If $0<\alpha<1, s \geq \alpha$, and $0<q \leq \infty$, then we have

$$
\|u\|_{C^{0, \alpha}(\Omega)} \leq C_{\alpha, s, q}\|u\|_{A^{s, p \alpha, s, q}(\Omega)} \quad \text { for } u \in A^{s, p_{\alpha, s}, q}(\Omega)
$$

since Lemmas 2.1 and 2.2 give $A^{s, p_{\alpha, s}, q}(\Omega) \hookrightarrow A^{s, p_{\alpha, s}, \infty}(\Omega) \hookrightarrow B^{s, p_{\alpha, s}, \infty}(\Omega) \hookrightarrow B^{\alpha, \infty, \infty}(\Omega) \simeq$ $C^{0, \alpha}(\Omega) \hookrightarrow \dot{C}^{0, \alpha}(\Omega)$. In view of Proposition 1.2 (i), Theorem 1.5 (i) immediately follows from what we have been calculating, that is, Theorem 4.1 and (4.1).

To prove Theorem 1.7(i), Lemmas 2.1 and 2.2 reduce the matters to the following proposition:

Proposition 4.2. Let $n \geq 2, \alpha \geq 1$, let $\Omega$ be a bounded domain in $\mathbb{R}^{n}$ satisfying the strong local Lipschitz condition, and $X(\Omega)=B^{\alpha, \infty, \infty}(\Omega)$. Assume that either (I) or (II)' holds. Then there exists a constant $C$ such that inequality (1.7) holds for all $u \in W_{0}^{1, n}(\Omega) \cap B^{\alpha, \infty, \infty}(\Omega)$ with $\|\nabla u\|_{L^{n}(\Omega)}=1$.

To prove it, we apply Theorem 1.5 (i).

\section{Proof of Proposition 4.2.}

Step 1. Consider the case $\lambda_{2} \geq 0$. Under assumption (I), choose $0<\gamma<1$ arbitrarily, and then

$$
\frac{\alpha}{\gamma} \lambda_{1}>\frac{\Lambda_{1}}{\gamma}, \quad \lambda_{2} \in \mathbb{R}
$$

Under assumption (II)', choose $0<\gamma<1$ such that $\lambda_{2} \geq \Lambda_{2} / \gamma$, and then

$$
\frac{\alpha}{\gamma} \lambda_{1}=\frac{\Lambda_{1}}{\gamma}, \quad \lambda_{2} \geq \frac{\Lambda_{2}}{\gamma}
$$

We apply Theorem 4.1 with replacing $\alpha$ by $\gamma$ to obtain

$$
\|u\|_{L^{\infty}(\Omega)}^{n /(n-1)} \leq \frac{\alpha}{\gamma} \lambda_{1} \ell\left(\|u\|_{\dot{C}^{0, r}(\Omega)}\right)+\lambda_{2} \ell \circ \ell\left(\|u\|_{\dot{C}^{0, r}(\Omega)}\right)+C_{\Omega, \alpha, \gamma, \lambda_{1}, \lambda_{2}}
$$

for $u \in W_{0}^{1, n}(\Omega) \cap \dot{C}^{0, \gamma}(\Omega)$ with $\|\nabla u\|_{L^{n}(\Omega)}=1$. On the other hand, Lemma 2.1 gives

$$
\|u\|_{C^{0, r}(\Omega)} \leq\|u\|_{C^{0, r}(\Omega)} \leq C_{\gamma}\|u\|_{B^{r, \infty, \infty}(\Omega)} .
$$


Combining (2.21), (4.4), and (4.5) yields

$$
\begin{aligned}
\|u\|_{L^{\infty}(\Omega)}^{n /(n-1)} \leq & \frac{\alpha}{\gamma} \lambda_{1} \ell\left(C_{\alpha, \gamma}\|u\|_{B^{\alpha, \infty}, \infty(\Omega)}^{\gamma / \alpha}\right) \\
& +\lambda_{2} \ell \circ \ell\left(C_{\alpha, \gamma}\|u\|_{B^{\alpha, \infty, \infty}(\Omega)}^{\gamma / \alpha}\right)+C_{\Omega, \alpha, \gamma, \lambda_{1}, \lambda_{2}} \\
\leq & \lambda_{1} \ell\left(2\left(1+C_{\alpha, \gamma}\|u\|_{B^{\alpha, \infty, \infty}(\Omega)}\right)\right) \\
& +\lambda_{2} \ell\left(\frac{\gamma}{\alpha} \log \left(2\left(1+C_{\alpha, \gamma}\|u\|_{B^{\alpha, \infty, \infty}(\Omega)}\right)\right)\right)+C_{\Omega, \alpha, \gamma, \lambda_{1}, \lambda_{2}} \\
\leq & \lambda_{1} \ell\left(\|u\|_{B^{\alpha, \infty, \infty}(\Omega)}\right)+\lambda_{2} \ell \circ \ell\left(\|u\|_{B^{\alpha, \infty, \infty}(\Omega)}\right)+C_{\Omega, \alpha, \gamma, \lambda_{1}, \lambda_{2}}
\end{aligned}
$$

for $u \in W_{0}^{1, n}(\Omega) \cap B^{\alpha, \infty, \infty}(\Omega)$ with $\|\nabla u\|_{L^{n}(\Omega)}=1$, and the assertion follows.

Step 2. Consider the remaining case $\lambda_{1}>\Lambda_{1} / \alpha$ and $\lambda_{2}<0$. We argue as in the proof of Lemma 5.4. Let $\delta=\lambda_{1} / 2-\Lambda_{1} /(2 \alpha)$. Note that $\delta>0$ and $\lambda_{1}-\delta>\Lambda_{1} / \alpha$. Since $\ell \circ \ell(s) / \ell(s) \rightarrow 0$ as $s \rightarrow \infty$, there exists a constant $C_{\delta}>0$ such that

$$
\ell \circ \ell(s) \leq-\frac{\delta}{\lambda_{2}} \ell(s)+C_{\delta} \text { for } s \geq 0
$$

We have from Step 1 that

$$
\|u\|_{L^{\infty}(\Omega)}^{n /(n-1)} \leq\left(\lambda_{1}-\delta\right) \ell\left(\|u\|_{B^{\alpha, \infty, \infty}(\Omega)}\right)+C_{\Omega, \alpha, \lambda_{1}, \delta}
$$

holds for $u \in W_{0}^{1, n}(\Omega) \cap B^{\alpha, \infty, \infty}(\Omega)$ with $\|\nabla u\|_{L^{n}(\Omega)}=1$. Then

$$
\|u\|_{L^{\infty}(\Omega)}^{n /(n-1)} \leq \lambda_{1} \ell\left(\|u\|_{B^{\alpha, \infty, \infty}(\Omega)}\right)+\lambda_{2} \ell \circ \ell\left(\|u\|_{B^{\alpha, \infty, \infty}(\Omega)}\right)+C_{\Omega, \alpha, \lambda_{1}, \lambda_{2}, \delta}
$$

holds for $u \in W_{0}^{1, n}(\Omega) \cap B^{\alpha, \infty, \infty}(\Omega)$ with $\|\nabla u\|_{L^{n}(\Omega)}=1$, and the assertion follows.

Remark 4.3. As is mentioned in the introduction, the power $r=n /(n-1)$ on the left-hand side of (1.7) is optimal (in the case $\alpha \geq 1$ ) in the sense that $r=n /(n-1)$ is the largest power for which there exist $\lambda_{1}$ and $C$ such that

$$
\|u\|_{L^{\infty}(\Omega)}^{r} \leq \lambda_{1} \ell\left(\|u\|_{B^{\alpha, \infty, \infty}(\Omega)}\right)+C
$$

can hold for all $u \in W_{0}^{1, n}(\Omega) \cap B^{\alpha, \infty, \infty}(\Omega)$ with $\|\nabla u\|_{L^{n}(\Omega)}=1$. Indeed, if $1 \leq r<n /(n-1)$, then for any $\lambda_{1}>0$, there exists a constant $C$ such that (4.10) holds for all $u \in W_{0}^{1, n}(\Omega) \cap B^{\alpha, \infty, \infty}(\Omega)$ with $\|\nabla u\|_{L^{n}(\Omega)}=1$. An argument similar to Proposition 4.2 works if we invoke the fact in [8, Remark 3.5] (for $0<\alpha<1$ ). Namely, the assertion for $\alpha \geq 1$ follows from the corresponding fact in the case $0<\alpha<1$. 


\section{Establishment of the Inequality (II)}

In this section, we will prove Theorem 1.9(i). In analogy with (4.1), if $1<\alpha<2, s \geq \alpha$ and $0<q \leq \infty$, then we have

$$
\|u\|_{\dot{C}^{1, \alpha-1}(\Omega)} \leq C_{\alpha, s, q}\|u\|_{A^{s, p \alpha, s, q}(\Omega)} \quad \text { for } u \in A^{s, p_{\alpha, s}, q}(\Omega) .
$$

By Proposition 2.4, it holds

$$
\|u\|_{\dot{C}_{\nabla}^{1, \alpha-1}(\Omega)} \leq C_{\alpha}\|u\|_{\dot{C}^{1, \alpha-1}(\Omega)} \quad \text { for } u \in \dot{C}^{1, \alpha-1}(\Omega)
$$

In view of (5.1), (5.2), and Proposition 1.2 (i), Theorem 1.9 (i) will have been proved once we establish the following theorem, which extends Theorem 4.1 to the case $1<\alpha \leq 2$.

Theorem 5.1. Let $n \geq 2,1<\alpha \leq 2$, and let $\Omega$ be a bounded domain in $\mathbb{R}^{n}$ and $X(\Omega)=\dot{C}_{\nabla}^{1, \alpha-1}(\Omega)$. Assume that either (I) or (II) holds. Then there exists a constant $C$ such that inequality (1.7) holds for all $u \in W_{0}^{1, n}(\Omega) \cap \dot{C}_{\nabla}^{1, \alpha-1}(\Omega) \cap C_{c}(\Omega)$ with $\|\nabla u\|_{L^{n}(\Omega)}=1$.

We argue as in [8] to prove Theorem 5.1.

In order to obtain our results, we examine a problem of minimizing $\|\nabla u\|_{L^{n}(\Omega)}^{n}$ with a unilateral constraint. Let $0<\tau \leq 1$. We consider the following minimizing problem:

$$
m\left[\Omega, h_{\tau}\right]=\inf \left\{\|\nabla u\|_{L^{n}\left(B_{1}\right)}^{n} ; u \in K\left[B_{1}, h_{\tau}\right]\right\}, \quad \quad\left(\mathrm{M} ; B_{1} ; h_{\tau}\right)
$$

where

$$
K\left[B_{1}, h_{\tau}\right]=\left\{u \in W_{0}^{1, n}\left(B_{1}\right) ; u \geq h_{\tau} \text { a.e. on } B_{1}\right\} .
$$

Here the obstacle function $h_{\tau}$ is given by

$$
h_{\tau}(x)=\tilde{h}_{\tau}(|x|)=1-\left(\frac{|x|}{T_{\tau}}\right)^{\alpha} \text { for } x \in \mathbb{R}^{n}
$$

where

$$
T_{\tau}=\tau\left(\alpha \log \frac{1}{\tau}+1\right)^{1 / \alpha}
$$

It is crucial to prove the following fact, which explicitly gives the minimizer $u_{\tau}^{\#}$ of the minimizing problem $\left(\mathrm{M} ; B_{1} ; h_{\tau}\right)$ with a parameter $0<\tau \leq 1$.

Then we can prove the following fact for $0<\alpha \leq 1$ as in [8]. Meanwhile it is also valid for $1<\alpha \leq 2$; the proof is completely identical. 
Lemma 5.2. Let $n \geq 2$ and $0<\alpha \leq 2$. For any $0<\tau \leq 1$, the unique minimizer $u_{\tau}^{\#}$ of $\left(\mathrm{M} ; B_{1} ; h_{\tau}\right)$ is given by

$$
u_{\tau}^{\#}(x)=\tilde{u}_{\tau}^{\#}(|x|)= \begin{cases}h_{\tau}(x) & \text { for } x \in \bar{B}_{\tau}, \\ \alpha\left(\frac{\tau}{T_{\tau}}\right)^{\alpha} \log \frac{1}{|x|} & \text { for } x \in B_{1} \backslash B_{\tau} .\end{cases}
$$

Remark 5.3. We can calculate the norms of $u_{\tau}^{\#}$ as

$$
\begin{gathered}
\left\|u_{\tau}^{\#}\right\|_{L^{\infty}\left(B_{1}\right)}=1, \quad\left\|\nabla u_{\tau}^{\#}\right\|_{L^{n}\left(B_{1}\right)}^{n}=\left(\frac{\alpha}{\Lambda_{1}}\right)^{n-1} \frac{\alpha \log (1 / \tau)+1 / n}{(\alpha \log (1 / \tau)+1)^{n}} \\
\left\|u_{\tau}^{\#}\right\|_{\dot{C}_{\nabla}^{1, \alpha-1}\left(B_{1}\right)}=\frac{\alpha 2^{2-\alpha}}{T_{\tau}^{\alpha}} .
\end{gathered}
$$

Although equalities (5.7) are straightforward and elementary, we will verify equality (5.8) in the appendix for the sake of completeness. We prove Theorem 5.1 by accepting (5.8).

In order to examine whether (1.7) holds or not, we may assume that $\lambda_{1} \geq 0$ and define

$$
\begin{gathered}
F_{\gamma}\left[u ; \lambda_{1}, \lambda_{2}\right]=\left(\frac{\|u\|_{L^{\infty}(\Omega)}}{\|\nabla u\|_{L^{n}(\Omega)}}\right)^{n /(n-1)}-\lambda_{1} \ell\left(r \frac{\|u\|_{\dot{C}_{\nabla}^{1, \alpha-1}(\Omega)}}{\|\nabla u\|_{L^{n}(\Omega)}}\right)-\lambda_{2} \ell \circ \ell\left(r \frac{\|u\|_{\dot{C}_{\nabla}^{1, \alpha-1}(\Omega)}}{\|\nabla u\|_{L^{n}(\Omega)}}\right) \\
\text { for } u \in W_{0}^{1, n}(\Omega) \cap \dot{C}_{\nabla}^{1, \alpha-1}(\Omega) \backslash\{0\}, r>0, \\
F\left[u ; \lambda_{1}, \lambda_{2}\right]=F_{1}\left[u ; \lambda_{1}, \lambda_{2}\right], \\
F^{*}\left[\lambda_{1}, \lambda_{2} ; \Omega\right]=\sup \left\{F\left[u ; \lambda_{1}, \lambda_{2}\right] ; u \in W_{0}^{1, n}(\Omega) \cap \dot{C}_{\nabla}^{1, \alpha-1}(\Omega) \cap C_{\mathrm{C}} \backslash(\Omega)\{0\}\right\}
\end{gathered}
$$

for $\lambda_{1} \geq 0, \lambda_{2} \in \mathbb{R}$. Note that

$$
F\left[c u ; \lambda_{1}, \lambda_{2}\right]=F\left[u ; \lambda_{1}, \lambda_{2}\right] \quad \forall c \in \mathbb{R} \backslash\{0\} .
$$

We also remark that

$$
F_{\gamma}\left[u ; \lambda_{1}, \lambda_{2}\right] \leq F\left[u ; \lambda_{1}, \lambda_{2}\right]+C_{\gamma, \lambda_{1}, \lambda_{2}} \text { for } u \in W_{0}^{1, n}(\Omega) \cap \dot{C}_{\nabla}^{1, \alpha-1}(\Omega) \backslash\{0\} .
$$


Indeed, since $\max \{\ell(s t), \ell(s+t)\} \leq \ell(s)+\ell(t)$ for $s, t \geq 0$, we have

$$
\begin{aligned}
F_{\gamma}\left[u ; \lambda_{1}, \lambda_{2}\right]-F\left[u ; \lambda_{1}, \lambda_{2}\right] & \\
= & \lambda_{1}\left(\ell\left(\frac{\|u\|_{\dot{C}_{\nabla}^{1, \alpha-1}(\Omega)}}{\|\nabla u\|_{L^{n}(\Omega)}}\right)-\ell\left(\gamma \frac{\|u\|_{\dot{C}_{\nabla}^{1, \alpha-1}(\Omega)}}{\|\nabla u\|_{L^{n}(\Omega)}}\right)\right) \\
& +\lambda_{2}\left(\ell \circ \ell\left(\frac{\|u\|_{\dot{C}_{\nabla}^{1, \alpha-1}(\Omega)}}{\|\nabla u\|_{L^{n}(\Omega)}}\right)-\ell \circ \ell\left(\gamma \frac{\|u\|_{\dot{C}_{\nabla}^{1, \alpha-1}(\Omega)}}{\|\nabla u\|_{L^{n}(\Omega)}}\right)\right) \\
\leq & \lambda_{1} \ell\left(\frac{1}{\gamma}\right)+\left|\lambda_{2}\right| \ell \circ \ell\left(\frac{1}{r^{\operatorname{sgn} \lambda_{2}}}\right) .
\end{aligned}
$$

Then under our new notations, Theorem 5.1 is equivalent to the following.

Lemma 5.4. Let $\Omega$ be a bounded domain in $\mathbb{R}^{n}$. Then the following hold.

(i) For any $\lambda_{1}>\Lambda_{1} / \alpha$ and $\lambda_{2} \in \mathbb{R}$, it holds $F^{*}\left[\lambda_{1}, \lambda_{2} ; \Omega\right]<\infty$.

(ii) For any $\lambda_{2} \geq \Lambda_{2} / \alpha$, it holds $F^{*}\left[\Lambda_{1} / \alpha, \lambda_{2} ; \Omega\right]<\infty$.

The aim of this section is to prove Lemma 5.4. Let us first reduce our problem on a general bounded domain $\Omega$ to that on the unit open ball $B_{1}$. We set

$$
\begin{gathered}
\widehat{K}=\left\{u \in W_{0}^{1, n}\left(B_{1}\right) \cap \dot{C}_{\nabla}^{1, \alpha-1}\left(B_{1}\right) ;\|u\|_{L^{\infty}\left(B_{1}\right)}=u(0)=1\right\}, \\
\widehat{F}^{*}\left[\lambda_{1}, \lambda_{2}\right]=\sup \left\{F\left[u ; \lambda_{1}, \lambda_{2}\right] ; u \in \widehat{K}\right\} \quad \text { for } \lambda_{1} \geq 0, \lambda_{2} \in \mathbb{R} .
\end{gathered}
$$

Proposition 5.5. Let $\Omega$ be a bounded domain in $\mathbb{R}^{n}$ and $\lambda_{1} \geq 0, \lambda_{2} \in \mathbb{R}$. If $\widehat{F}^{*}\left[\lambda_{1}, \lambda_{2}\right]<\infty$, then it holds $F^{*}\left[\lambda_{1}, \lambda_{2} ; \Omega\right]<\infty$.

Proof. Let $u \in W_{0}^{1, n}(\Omega) \cap \dot{C}_{\nabla}^{1, \alpha-1}(\Omega) \cap C_{\mathrm{c}}(\Omega) \backslash\{0\}$. Suppose that $|u|$ attains its maximum at a point $z_{u} \in \Omega$. Define a function $v_{u}: B_{1} \rightarrow \mathbb{R}$ by

$$
v_{u}(x)= \begin{cases}\frac{\operatorname{sgn} u\left(z_{u}\right)}{\|u\|_{L^{\infty}(\Omega)}} u\left(d_{\Omega} x+z_{u}\right) & \text { if } d_{\Omega} x+z_{u} \in \Omega, \\ 0 & \text { otherwise }\end{cases}
$$

for $x \in B_{1}$, where $d_{\Omega}=\operatorname{diam} \Omega=\sup \{|x-y| ; x, y \in \Omega\}$. Then we have $v_{u} \in \widehat{K}$ and

$$
\left\|\nabla v_{u}\right\|_{L^{n}\left(B_{1}\right)}=\frac{\|\nabla u\|_{L^{n}(\Omega)}}{\|u\|_{L^{\infty}(\Omega)}}, \quad\left\|v_{u}\right\|_{\dot{C}_{\nabla}^{1, \alpha-1}\left(B_{1}\right)}=d_{\Omega}^{\alpha} \frac{\|u\|_{\dot{C}_{\nabla}^{1, \alpha-1}(\Omega)}}{\|u\|_{L^{\infty}(\Omega)}}
$$


by the dilation property and translation invariance. Applying (5.12), we have

$$
\begin{aligned}
F\left[u ; \lambda_{1}, \lambda_{2}\right] & =F_{1 / d_{\Omega}^{\alpha}}\left[v_{u} ; \lambda_{1}, \lambda_{2}\right]=F\left[v_{u} ; \lambda_{1}, \lambda_{2}\right]+C_{\Omega, \alpha, \lambda_{1}, \lambda_{2}} \\
& \leq \widehat{F}^{*}\left[\lambda_{1}, \lambda_{2}\right]+C_{\Omega, \alpha, \lambda_{1}, \lambda_{2}} \quad \text { for } u \in W_{0}^{1, n}(\Omega) \cap \dot{C}_{\nabla}^{1, \alpha-1}(\Omega) \cap C_{\mathrm{c}}(\Omega) \backslash\{0\}
\end{aligned}
$$

Therefore, if $\widehat{F}^{*}\left[\lambda_{1}, \lambda_{2}\right]<\infty$, then $F^{*}\left[\lambda_{1}, \lambda_{2} ; \Omega\right]<\infty$.

For $\kappa>0$ and $\mu_{1}, \mu_{2} \geq 0$, define

$$
\begin{aligned}
G_{\kappa}\left(s ; \mu_{1}, \mu_{2}\right)= & \left(\frac{(s+1)^{n}}{s+1 / n}\right)^{1 /(n-1)}-\mu_{1} \ell\left(\frac{\kappa e^{s}}{(s+1 / n)^{1 / n}}\right) \\
& -\frac{\mu_{2}}{n} \ell \circ \ell\left(\frac{\kappa e^{s}}{(s+1 / n)^{1 / n}}\right) \text { for } s \geq 0 .
\end{aligned}
$$

As we will see just below, $G_{\kappa}\left(s ; \mu_{1}, \mu_{2}\right)$ majorizes $\widehat{F}^{*}\left[\lambda_{1}, \lambda_{2}\right]$. The idea of the proof of Proposition 5.6 is essentially due to [16].

Proposition 5.6. For any $\lambda_{1} \geq 0$ and $\lambda_{2} \in \mathbb{R}$, it holds

$$
\widehat{F}^{*}\left[\lambda_{1},\left(\lambda_{2}\right)_{+}\right] \leq \frac{\Lambda_{1}}{\alpha} \sup _{s \geq 0} G_{\left(\Lambda_{1} / \alpha\right)^{1-1 / n}}\left(s ; \frac{\alpha}{\Lambda_{1}} \lambda_{1}, \frac{\alpha}{\Lambda_{2}} \lambda_{2}\right)_{+}
$$

Proof. (a) We claim that $\widehat{K}$ is partitioned into $\left\{\widehat{K}_{\tau}\right\}_{0<\tau \leq 1}$ :

$$
\widehat{K}=\coprod_{0<\tau \leq 1} \widehat{K}_{\tau}
$$

where

$$
\widehat{K}_{\tau}=\left\{u \in K\left[B_{1}, h_{\tau}\right] \cap \dot{C}_{\nabla}^{1, \alpha-1}\left(B_{1}\right) ;\|u\|_{\dot{C}_{\nabla}^{1, \alpha-1}\left(B_{1}\right)}=\frac{1}{T_{\tau}^{\alpha}},\|u\|_{L^{\infty}\left(B_{1}\right)}=u(0)=1\right\} .
$$

It is trivial that $\widehat{K}_{\tau} \subset \widehat{K}$ for all $0<\tau \leq 1$. Conversely, for any $u \in \widehat{K}$, we have

$$
\|\nabla u\|_{L^{\infty}\left(B_{1}\right)} \geq 1
$$

Indeed, for $x \in \partial B_{1}=S^{n-1}$, we see that

$$
1=u(0)-u(x)=-\int_{0}^{1} x \cdot[\nabla u](t x) d t \leq|x| \int_{0}^{1}|[\nabla u](t x)| d t \leq\|\nabla u\|_{L^{\infty}\left(B_{1}\right)} .
$$


Therefore, since $|\nabla u|$ is continuous, $|\nabla u|$ attains its maximum at some $x_{0} \in B_{1}$. Since $u$ attains its maximum at the origin, the gradient vanishes there. Hence we have

$$
\|u\|_{\dot{C}_{\nabla}^{1, \alpha-1}\left(B_{1}\right)}=\|\nabla u\|_{\dot{C}^{0, \alpha-1}\left(B_{1} ; \mathbb{R}^{n}\right)} \geq \frac{\left|\nabla u\left(x_{0}\right)-\nabla u(0)\right|}{\left|x_{0}-0\right|^{\alpha-1}}=\frac{\left|\nabla u\left(x_{0}\right)\right|}{\left|x_{0}\right|^{\alpha-1}} \geq 1
$$

The triangle inequality for integrals yields

$$
\begin{aligned}
u(x) & =1+u(x)-u(0) \\
& =1+\int_{0}^{1} x \cdot[\nabla u](t x) d t \geq 1-|x| \int_{0}^{1}|[\nabla u](t x)| d t \\
& =1-|x|^{\alpha} \int_{0}^{1} t^{\alpha-1} \frac{|[\nabla u](t x)-\nabla u(0)|}{|t x-0|^{\alpha-1}} d t .
\end{aligned}
$$

From the definition of the seminorm, we obtain

$$
\begin{aligned}
u(x) & \geq 1-\frac{1}{\alpha}\|\nabla u\|_{\dot{C}^{0, \alpha-1}\left(B_{1} ; \mathbb{\mathbb { R } ^ { n }}\right)}|x|^{\alpha} \geq 1-\|\nabla u\|_{\dot{C}^{0, \alpha-1}\left(B_{1} ; \mathbb{R}^{n}\right)}|x|^{\alpha} \\
& =1-\|u\|_{\dot{C}_{\nabla}^{1, \alpha-1}\left(B_{1}\right)}|x|^{\alpha} \quad \text { for } x \in \bar{B}_{1} .
\end{aligned}
$$

Then, $u \in \widehat{K}_{\tau}$ with $1 / T_{\tau}^{\alpha}=\|u\|_{\dot{C}_{\nabla}^{1, \alpha-1}\left(B_{1}\right)} \geq 1$, and hence we obtain (5.20).

(b) Next we show that

$$
F\left[u ; \lambda_{1},\left(\lambda_{2}\right)_{+}\right] \leq F_{\alpha 2^{2-\alpha}}\left[u_{\tau}^{\#} ; \lambda_{1}, \lambda_{2}\right]_{+} \text {for } u \in \widehat{K}_{\tau} .
$$

Here $F_{\alpha 2^{2-\alpha}}$ is given by (5.9) with $\gamma=\alpha 2^{2-\alpha}$. Note that $\|\nabla u\|_{L^{n}\left(B_{1}\right)} \geq\left\|\nabla u_{\tau}^{\#}\right\|_{L^{n}\left(B_{1}\right)}$ for all $u \in$ $K\left[B_{1}, h_{\tau}\right]$. We also remark that $\left\|u_{\tau}^{\#}\right\|_{\dot{C}_{\nabla}^{1, \alpha-1}\left(B_{1}\right)}=\alpha 2^{2-\alpha} / T_{\tau}^{\alpha}$ and $\left\|u_{\tau}^{\#}\right\|_{L^{\infty}\left(B_{1}\right)}=u_{\tau}^{\#}(0)=1$. Since the functions

$$
(0, \infty) \ni s \longmapsto s^{n /(n-1)} \ell\left(\frac{1}{s}\right) \in(0, \infty), \quad(0, \infty) \ni s \longmapsto s^{n /(n-1)} \ell \circ \ell\left(\frac{1}{s}\right) \in(0, \infty)
$$


are both increasing, we have

$$
\begin{aligned}
\|\nabla u\|_{L^{n}\left(B_{1}\right)}^{n /(n-1)} F\left[u ; \lambda_{1},\left(\lambda_{2}\right)_{+}\right]= & 1-\lambda_{1}\|\nabla u\|_{L^{n}\left(B_{1}\right)}^{n /(n-1)} \ell\left(\frac{1}{T_{\tau}^{\alpha}} \frac{1}{\|\nabla u\|_{L^{n}\left(B_{1}\right)}}\right) \\
& -\left(\lambda_{2}\right)_{+}\|\nabla u\|_{L^{n}\left(B_{1}\right)}^{n /(n-1)} \ell \circ \ell\left(\frac{1}{T_{\tau}^{\alpha}} \frac{1}{\|\nabla u\|_{L^{n}\left(B_{1}\right)}}\right) \\
\leq & 1-\lambda_{1}\left\|\nabla u_{\tau}^{\#}\right\|_{L^{n}\left(B_{1}\right)}^{n /(n-1)} \ell\left(\frac{1}{T_{\tau}^{\alpha}} \frac{1}{\left\|\nabla u_{\tau}^{\#}\right\|_{L^{n}\left(B_{1}\right)}}\right) \\
& -\lambda_{2}\left\|\nabla u_{\tau}^{\#}\right\|_{L^{n}\left(B_{1}\right)}^{n /(n-1)} \ell \circ \ell\left(\frac{1}{T_{\tau}^{\alpha}} \frac{1}{\left\|\nabla u_{\tau}^{\#}\right\|_{L^{n}\left(B_{1}\right)}}\right) \\
= & \left\|\nabla u_{\tau}^{\#}\right\|_{L^{n}\left(B_{1}\right)}^{n /(n-1)} F_{\alpha 2^{2-\alpha}}\left[u_{\tau}^{\#} ; \lambda_{1}, \lambda_{2}\right] \\
\leq & \|\nabla u\|_{L^{n}\left(B_{1}\right)}^{n /(n-1)} F_{\alpha 2^{2-\alpha}}\left[u_{\tau}^{\#} ; \lambda_{1}, \lambda_{2}\right]_{+} \quad \text { for } u \in \widehat{K}_{\tau},
\end{aligned}
$$

which implies (5.27).

(c) It follows from Remark 5.3 that

$$
F_{\alpha 2^{2-\alpha}}\left[u_{\tau}^{\#} ; \lambda_{1}, \lambda_{2}\right]=\frac{\Lambda_{1}}{\alpha} G_{\alpha 2^{2-\alpha}\left(\Lambda_{1} / \alpha\right)^{1-1 / n}}\left(\alpha \log \frac{1}{\tau} ; \frac{\alpha}{\Lambda_{1}} \lambda_{1}, \frac{\alpha}{\Lambda_{2}} \lambda_{2}\right) \quad \text { for } 0<\tau \leq 1
$$

Combining (5.20)-(5.30) yields

$$
\begin{aligned}
\sup _{u \in \widehat{K}} F\left[u ; \lambda_{1},\left(\lambda_{2}\right)_{+}\right] & =\sup _{0<\tau \leq 1}\left(\sup _{u \in \widehat{K}_{\tau}} F\left[u ; \lambda_{1},\left(\lambda_{2}\right)_{+}\right]\right) \\
& \leq \sup _{0<\tau \leq 1}\left(\sup _{u \in \widehat{K}_{\tau}} F_{\alpha 2^{2-\alpha}}\left[u ; \lambda_{1},\left(\lambda_{2}\right)_{+}\right]\right) \\
& \leq \sup _{0<\tau \leq 1} F_{\alpha 2^{2-\alpha}}\left[u_{\tau}^{\#} ; \lambda_{1}, \lambda_{2}\right]_{+} \\
& =\frac{\Lambda_{1}}{\alpha} \sup _{0<\tau \leq 1} G_{\alpha 2^{2-\alpha}\left(\Lambda_{1} / \alpha\right)^{1-1 / n}}\left(\alpha \log \frac{1}{\tau} ; \frac{\alpha}{\Lambda_{1}} \lambda_{1}, \frac{\alpha}{\Lambda_{2}} \lambda_{2}\right)_{+} \\
& =\frac{\Lambda_{1}}{\alpha} \sup _{s \geq 0} G_{\alpha 2^{2-\alpha}\left(\Lambda_{1} / \alpha\right)^{1-1 / n}}\left(s ; \frac{\alpha}{\Lambda_{1}} \lambda_{1}, \frac{\alpha}{\Lambda_{2}} \lambda_{2}\right)_{+},
\end{aligned}
$$

which implies (5.19).

The following lemma describes the behavior of the function $G_{\kappa}\left(s ; \mu_{1}, \mu_{2}\right)$ as $s \rightarrow \infty$, which plays an essential role for proving Lemma 5.4. Here we invoked the result from [8, Lemma 3.4]. 
Lemma 5.7. Let $\kappa>0$.

(i) If $\mu_{1}>1, \mu_{2} \in \mathbb{R}$, or $\mu_{1}=1, \mu_{2}>1$, then $G_{\kappa}\left(s ; \mu_{1}, \mu_{2}\right) \rightarrow-\infty$ as $s \rightarrow \infty$.

(ii) $G_{\kappa}(s ; 1,1)$ is decreasing if $s$ is sufficiently large and tends to a finite limit $\widehat{G}_{\kappa}$ as $s \rightarrow \infty$.

(iii) If $\mu_{1}<1, \mu_{2} \in \mathbb{R}$, or $\mu_{1}=1, \mu_{2}<1$, then $G_{\kappa}\left(s ; \mu_{1}, \mu_{2}\right) \rightarrow \infty$ as $s \rightarrow \infty$.

We now show Lemma 5.4 by using Proposition 5.6 and Lemma 5.7. We divide assertion (i) in Lemma 5.4 into the following two assertions for the sake of convenience.

(i-1) For any $\lambda_{1}>\Lambda_{1} / \alpha$ and $\lambda_{2} \geq 0$, it holds $F^{*}\left[\lambda_{1}, \lambda_{2} ; \Omega\right]<\infty$;

(i-2) For any $\lambda_{1}>\Lambda_{1} / \alpha$ and $\lambda_{2}<0$, it holds $F^{*}\left[\lambda_{1}, \lambda_{2} ; \Omega\right]<\infty$.

Proof of Lemma 5.4. (a) First we show assertions (i-1) and (ii). We take $\mu_{1}=\alpha \lambda_{1} / \Lambda_{1}, \mu_{2}=$ $\alpha \lambda_{2} / \Lambda_{2}$, and $s=\alpha \log (1 / \tau)$. By virtue of Proposition 5.5, assertions (i-1) and (ii) follow from Lemma 5.7 (i) and (ii), respectively.

(b) Next we show assertion (i-2). Let $\delta=\lambda_{1} / 2-\Lambda_{1} /(2 \alpha)$. Note that $\delta>0$ and $\lambda_{1}-\delta>$ $\Lambda_{1} / \alpha$. We have from (a) that $F^{*}\left[\lambda_{1}-\delta, 0 ; \Omega\right]<\infty$. Applying (4.7), we have

$$
\begin{aligned}
F\left[u ; \lambda_{1}, \lambda_{2}\right]= & F\left[u ; \lambda_{1}-\delta, 0\right]-\lambda_{2}\left(\frac{\delta}{\lambda_{2}} \ell\left(\frac{\|u\|_{\dot{C}_{\nabla}^{1, \alpha-1}(\Omega)}}{\|\nabla u\|_{L^{n}(\Omega)}}\right)+\ell \circ \ell\left(\frac{\|u\|_{\dot{C}_{\nabla}^{1, \alpha-1}(\Omega)}}{\|\nabla u\|_{L^{n}(\Omega)}}\right)\right) \\
& \leq F^{*}\left[\lambda_{1}-\delta, 0 ; \Omega\right]-\lambda_{2} C_{\delta} \\
& <\infty \text { for } u \in W_{0}^{1, n}(\Omega) \cap \dot{C}_{\nabla}^{1, \alpha-1}(\Omega) \cap C_{\mathrm{c}}(\Omega) \backslash\{0\},
\end{aligned}
$$

and the assertion follows.

Thus we have proved Theorem 5.1.

\section{Appendices}

In this section, we carry out elementary calculi which we omitted in Sections 2 and 5.

\section{A. On the Space $\dot{C}^{1, \alpha-1}\left(\mathbb{R}^{n}\right)$}

First, we prove Proposition 2.3. Let $1<\alpha<2$, and let $\Omega$ be a domain in $\mathbb{R}^{n}$. Then we will prove that there exists $C_{\alpha}>0$ such that

$$
\|u\|_{\dot{C}_{\nabla}^{1, \alpha-1}(\Omega)} \leq C_{\alpha}\|u\|_{\dot{C}^{1, \alpha-1}(\Omega)} \quad \text { for } u \in \dot{C}^{1, \alpha-1}(\Omega)
$$

Proof of Proposition 2.3. By putting $y=x+h$ into (2.4), we deduce

$$
\left|u(x+h)-2 u\left(x+\frac{1}{2} h\right)+u(x)\right| \leq|h|^{\alpha}\|u\|_{\dot{C}^{1, \alpha-1}\left(\mathbb{R}^{n}\right)} .
$$


We choose an auxiliary radial function $\phi \in C_{\mathrm{c}}^{\infty}\left(\mathbb{R}^{n}\right)$ with integral 1 . Note that

$$
\int_{\mathbb{R}^{n}} \frac{\partial \phi}{\partial x_{i}}(x) d x=\int_{\mathbb{R}^{n}} \frac{\partial^{2} \phi}{\partial x_{i} \partial x_{j}}(x) d x=0 \quad \text { for } i, j \in\{1, \ldots, n\}
$$

We define $u_{k}$ by

$$
u_{k}(x)=2^{n k} \int_{\mathbb{R}^{n}} u(y) \phi\left(2^{k}(x-y)\right) d y \quad \text { for } k \in \mathbb{Z}
$$

Note that $u_{k} \rightarrow u$ locally uniformly in $\mathbb{R}^{n}$ as $k \rightarrow \infty$. Then we have

$$
\begin{aligned}
\frac{\partial^{2} u_{k}}{\partial x_{i} \partial x_{j}}(x) & =2^{(n+2) k} \int_{\mathbb{R}^{n}} u(y) \frac{\partial^{2} \phi}{\partial x_{i} \partial x_{j}}\left(2^{k}(x-y)\right) d y \\
& =2^{2 k} \int_{\mathbb{R}^{n}} u\left(x-\frac{1}{2^{k}} y\right) \frac{\partial^{2} \phi}{\partial x_{i} \partial x_{j}}(y) d y .
\end{aligned}
$$

Since $\phi$ is even, a change of variables $y \mapsto-y$ yields

$$
\frac{\partial^{2} u_{k}}{\partial x_{i} \partial x_{j}}(x)=2^{2 k} \int_{\mathbb{R}^{n}} u\left(x+\frac{1}{2^{k}} y\right) \frac{\partial^{2} \phi}{\partial x_{i} \partial x_{j}}(y) d y .
$$

Because of (A.3), we have

$$
\frac{\partial^{2} u_{k}}{\partial x_{i} \partial x_{j}}(x)=2^{2 k} \int_{\mathbb{R}^{n}}\left(u\left(x+\frac{1}{2^{k}} y\right)-2 u(x)+u\left(x-\frac{1}{2^{k}} y\right)\right) \frac{\partial^{2} \phi}{\partial x_{i} \partial x_{j}}(y) d y .
$$

It follows from (A.2) that

$$
\left|\frac{\partial^{2} u_{k}}{\partial x_{i} \partial x_{j}}(x)\right| \leq C_{\alpha} 2^{(2-\alpha) k}\|u\|_{C^{1, \alpha-1}\left(\mathbb{R}^{n}\right)} .
$$

Meanwhile we have

$$
\frac{\partial u_{k}}{\partial x_{i}}(x)=2^{k} \int_{\mathbb{R}^{n}} u\left(x+\frac{1}{2^{k}} y\right) \frac{\partial \phi}{\partial x_{i}}(y) d y
$$

which yields

$$
\frac{\partial u_{k+1}}{\partial x_{i}}(x)-\frac{\partial u_{k}}{\partial x_{i}}(x)=2^{k} \int_{\mathbb{R}^{n}}\left(2 u\left(x+\frac{1}{2^{k+1}} y\right)-u\left(x+\frac{1}{2^{k}} y\right)\right) \frac{\partial \phi}{\partial x_{i}}(y) d y
$$


Because of (A.3), we have

$$
\frac{\partial u_{k+1}}{\partial x_{i}}(x)-\frac{\partial u_{k}}{\partial x_{i}}(x)=-2^{k} \int_{\mathbb{R}^{n}}\left(u\left(x+\frac{1}{2^{k}} y\right)-2 u\left(x+\frac{1}{2^{k+1}} y\right)+u(x)\right) \frac{\partial \phi}{\partial x_{i}}(y) d y .
$$

It follows from (A.2) that

$$
\left|\frac{\partial u_{k+1}}{\partial x_{i}}(x)-\frac{\partial u_{k}}{\partial x_{i}}(x)\right| \leq \frac{C}{2^{(\alpha-1) k}}\|u\|_{\dot{C}^{1, \alpha-1}\left(\mathbb{R}^{n}\right)}
$$

and hence

$$
u_{k}=u_{0}+\sum_{l=1}^{k}\left(u_{l}-u_{l-1}\right)
$$

converges in $C_{\text {loc }}^{1}\left(\mathbb{R}^{n}\right)$ as $k \rightarrow \infty$. In particular, $u \in C^{1}\left(\mathbb{R}^{n}\right)$. Now we fix $x, y \in \mathbb{R}^{n}$ arbitrarily. Since $1<\alpha<2$, we have from (A.8) that

$$
\left|\nabla u_{-k}(x)-\nabla u_{-k}(y)\right| \leq \frac{C_{\alpha}}{2^{(2-\alpha) k}}\|u\|_{\dot{C}^{1, \alpha-1}\left(\mathbb{R}^{n}\right)}|x-y|^{\alpha-1} \longrightarrow 0 \quad \text { as } k \longrightarrow \infty .
$$

We apply inequalities (A.8) and (A.12) to conclude

$$
\begin{aligned}
& \left|\nabla u_{k}(x)-\nabla u_{k}(y)-\nabla u_{-k}(x)+\nabla u_{-k}(y)\right| \\
& \quad \leq \sum_{l=-k+1}^{k}\left|\nabla u_{l}(x)-\nabla u_{l}(y)-\nabla u_{l-1}(x)+\nabla u_{l-1}(y)\right| \\
& \quad \leq C_{\alpha} \sum_{l=-\infty}^{\infty} \min \left\{|x-y| 2^{(2-\alpha) l}, \frac{1}{2^{(\alpha-1) l}}\right\}\|u\|_{C^{1, \alpha-1}\left(\mathbb{R}^{n}\right)} \\
& \quad \leq C_{\alpha}\|u\|_{\mathcal{C}^{1, \alpha-1}\left(\mathbb{R}^{n}\right)}|x-y|^{\alpha-1} .
\end{aligned}
$$

Since $u_{k} \rightarrow u, \nabla u_{k} \rightarrow \nabla u$ locally uniformly in $\mathbb{R}^{n}$ as $k \rightarrow \infty$, the assertion follows from (A.14).

When we defined $u_{k}$ by (A.4), we used the continuity of $u$, or more precisely, we used the local integrability of $u$. As is announced in Section 2, this type of assumption is absolutely necessary.

Proposition A.1. Let $\alpha>0$. Then there exists a discontinuous function $u: \mathbb{R}^{n} \rightarrow \mathbb{R}$ satisfying

$$
\|u\|_{\dot{C}^{1, \alpha-1}\left(\mathbb{R}^{n}\right)}=\sup _{\substack{x, y \in \mathbb{R}^{n} \\ x \neq y}} \frac{|u(x)-2 u((x+y) / 2)+u(y)|}{|x-y|^{\alpha}}=0,
$$

that is, $u(x)-2 u((x+y) / 2)+u(y)=0$ for all $x, y \in \mathbb{R}^{n}$. 
Proof. Choose a Hamel basis, that is, a $\mathbb{Q}$-basis $\left\{\xi_{\Lambda}\right\}_{\Lambda \in \Lambda}$ of $\mathbb{R}^{n}$. If necessary, we can assume that

$$
e_{j}=(0, \ldots, 0, \stackrel{j \text { th }}{1}, 0, \ldots, 0), \quad e=\sqrt{2} e_{1} \in\left\{\xi_{\lambda}\right\}_{\lambda \in \Lambda} .
$$

Accordingly, we fix a collection of real numbers $Z=\left\{z_{\lambda}\right\}_{\lambda \in \Lambda} \subset \mathbb{R}$. Then define a function $u_{Z}: \mathbb{R}^{n} \rightarrow \mathbb{R}$ so that

$$
u_{Z}\left(\sum_{\lambda \in \Lambda_{0}} q_{\lambda} \xi_{\lambda}\right)=\sum_{\lambda \in \Lambda_{0}} q_{\lambda} z_{\lambda}
$$

for all finite subsets $\Lambda_{0} \subset \Lambda$ and $\left\{q_{\lambda}\right\}_{\lambda \in \Lambda_{0}} \subset \mathbb{Q}$. From definition (A.18) we can verify that $\left\|u_{Z}\right\|_{C^{1, \alpha-1}\left(\mathbb{R}^{n}\right)}=0$.

Now we have freedom to choose $Z=\left\{z_{\lambda}\right\}_{\lambda \in \Lambda}$. If $u_{Z}$ is continuous, then we have

$$
u_{Z}(x)=\lim _{\substack{y \rightarrow x \\ y \in \mathbb{Q}^{n}}} u_{Z}(y)=\lim _{\substack{y=\left(y_{1}, y_{2}, \ldots, y_{n}\right) \rightarrow x \\ y \in \mathbb{Q}^{n}}} \sum_{j=1}^{n} y_{j} u_{Z}\left(e_{j}\right)=\sum_{j=1}^{n} x_{j} u_{Z}\left(e_{j}\right)
$$

for all $x=\left(x_{1}, x_{2}, \ldots, x_{n}\right) \in \mathbb{R}^{n}$. Hence $u_{Z}$ is continuous if and only if $u_{Z}$ is $\mathbb{R}$-linear. Keeping this in mind, if we choose $\left\{z_{\lambda}\right\}_{\lambda \in \Lambda}$ so that $u_{Z}(e)=1, u_{Z}\left(e_{j}\right)=0$ for each $j \in\{1,2, \ldots, n\}$, then $u_{Z}$ is the desired discontinuous function satisfying $\left\|u_{Z}\right\|_{C^{1, \alpha-1}\left(\mathbb{R}^{n}\right)}=0$.

\section{B. Proof of Equality (5.8)}

We are left with verifying equality (5.8) according to definition (2.10).

Lemma B.1. Let $0<\tau \leq 1$ and $1<\alpha \leq 2$. Then it holds $\nabla u_{\tau}^{\#} \in \dot{C}^{0, \alpha-1}\left(B_{1} ; \mathbb{R}^{n}\right)$ and

$$
\left\|\nabla u_{\tau}^{\#}\right\|_{C^{0, \alpha-1}\left(B_{1} ; \mathbb{R}^{n}\right)}=\frac{\alpha 2^{2-\alpha}}{T_{\tau}^{\alpha}}
$$

Let us define

$$
\mathbf{f}(x)= \begin{cases}\frac{x}{|x|^{2-\alpha}} & \text { for } x \in \bar{B}_{1} \\ \frac{x}{|x|^{2}} & \text { for } x \in \mathbb{R}^{n} \backslash B_{1}\end{cases}
$$

In view of (5.6), we have

$$
\nabla u_{\tau}^{\#}(x)=-\frac{\alpha \tau^{\alpha-1}}{T_{\tau}^{\alpha}} \mathbf{f}\left(\frac{x}{\tau}\right) \text { for } x \in B_{1}
$$

and hence $\left\|\nabla u_{\tau}^{\#}\right\|_{\dot{C}^{0, \alpha-1}\left(B_{1} ; \mathbb{R}^{n}\right)}=\left(\alpha / T_{\tau}^{\alpha}\right)\left\|\mathbf{f}_{B_{1 / \tau}}\right\|_{\dot{C}^{0, \alpha-1}\left(B_{1 / \tau} ; \mathbb{R}^{n}\right)}$. Then Lemma B.1 is equivalent to the following. 
Lemma B.2. Let $R \geq 1$ and $1<\alpha \leq 2$. Then it holds $\left.\mathbf{f}\right|_{B_{R}} \in \dot{C}^{0, \alpha-1}\left(B_{R} ; \mathbb{R}^{n}\right)$ and

$$
\left\|\left.\mathbf{f}\right|_{B_{R}}\right\|_{\dot{C}^{0, \alpha-1}\left(B_{R} ; \mathbb{R}^{n}\right)}=2^{2-\alpha} .
$$

To prove Lemma B.2, we need to establish some propositions.

Proposition B.3. Let $1<\alpha \leq 2,0 \leq \theta<1, \eta>0$, and

$$
\Phi_{\theta, \eta}(t)=\frac{1+\eta^{2}-2 \eta t}{\left(1+\theta^{2}-2 \theta t\right)^{\alpha-1}} \quad \text { for }-1 \leq t \leq 1
$$

Then one has

$$
\max _{-1 \leq t \leq 1} \Phi_{\theta, \eta}(t)=\max \left\{\Phi_{\theta, \eta}(-1), \Phi_{\theta, \eta}(1)\right\}
$$

Furthermore, if $\theta \leq \eta \leq 1$ or $\theta \leq 1 / \eta \leq 1$, then

$$
\max _{-1 \leq t \leq 1} \Phi_{\theta, \eta}(t)=\Phi_{\theta, \eta}(-1)
$$

Proof. Since

$$
\left(1+\theta^{2}-2 \theta t\right)^{\alpha} \frac{d \Phi_{\theta, \eta}}{d t}(t)=2\left(2(2-\alpha) \theta \eta t-\eta\left(1+\theta^{2}\right)+(\alpha-1) \theta\left(1+\eta^{2}\right)\right)
$$

we have

$$
\frac{d}{d t}\left[\left(1+\theta^{2}-2 \theta t\right)^{\alpha} \frac{d \Phi_{\theta, \eta}}{d t}\right](t)=4(2-\alpha) \theta \eta \geq 0 \quad \text { for }-1<t<1
$$

Hence the maximum principle shows that $\Phi_{\theta, \eta}$ attains its maximum on $\partial[-1,1]=\{-1,1\}$.

To prove the latter assertion, we will show that $\Phi_{\theta, \eta}(-1) \geq \Phi_{\theta, \eta}(1)$. Since $[0,1) \ni s \mapsto$ $(1-s) /(1+s) \in(0,1]$ is decreasing, we have

$$
\begin{gathered}
\frac{1-\eta}{1+\eta} \leq \frac{1-\theta}{1+\theta} \leq\left(\frac{1-\theta}{1+\theta}\right)^{\alpha-1} \leq 1 \quad \text { for } \theta \leq \eta \leq 1 \\
\frac{\eta-1}{\eta+1}=\frac{1-1 / \eta}{1+1 / \eta} \leq \frac{1-\theta}{1+\theta} \leq\left(\frac{1-\theta}{1+\theta}\right)^{\alpha-1} \leq 1 \quad \text { for } \theta \leq \frac{1}{\eta} \leq 1 .
\end{gathered}
$$

These inequalities imply

$$
\left(\Phi_{\theta, \eta}(-1)\right)^{1 / 2}=\frac{1+\eta}{(1+\theta)^{\alpha-1}} \geq \frac{|1-\eta|}{(1-\theta)^{\alpha-1}}=\left(\Phi_{\theta, \eta}(1)\right)^{1 / 2}
$$


provided that $\theta \leq \eta \leq 1$ or $\theta \leq 1 / \eta \leq 1$. The proof is now completed.

One can easily show the following proposition by a direct calculation.

Proposition B.4. Let $1<\alpha \leq 2,0<r \leq 1$, and

$$
\begin{gathered}
g_{\alpha}(t)=\frac{1+t^{\alpha-1}}{(1+t)^{\alpha-1}} \quad \text { for } 0 \leq t \leq 1, \\
\tilde{g}_{\alpha}(s)=\frac{(1+s)^{2-\alpha}}{s} \quad \text { for } s>0 \\
G_{\alpha, r}(\rho)=\frac{1+\rho r^{\alpha-1}}{\rho(\rho+r)^{\alpha-1}} \quad \text { for } \rho \geq 1 .
\end{gathered}
$$

Then the function $g_{\alpha}$ is increasing on $[0,1]$, the function $\tilde{g}_{\alpha}$ is decreasing on $(0, \infty)$, the function $G_{\alpha, r}$ is decreasing on $[1, \infty)$, and hence

$$
\begin{gathered}
g_{\alpha}(t) \leq 2^{2-\alpha} \quad \text { for } 0 \leq t \leq 1, \\
\tilde{g}_{\alpha}(s) \leq 2^{2-\alpha} \quad \text { for } s \geq 1, \\
G_{\alpha, r}(\rho) \leq 2^{2-\alpha} \quad \text { for } \rho \geq 1 .
\end{gathered}
$$

We now turn to proving Lemma B.2.

Proof of Lemma B.2. If we choose $0<r<1$, then it is easy to see that

$$
\left\|\left.\mathbf{f}\right|_{B_{R}}\right\|_{\dot{C}^{0, \alpha-1}\left(B_{R} ; \mathbb{R}^{n}\right)} \geq \frac{\left|\mathbf{f}\left(r e_{1}\right)-\mathbf{f}\left(-r e_{1}\right)\right|}{\left|r e_{1}-\left(-r e_{1}\right)\right|^{\alpha-1}}=2^{2-\alpha}
$$

We will use the substitutions $x=r e_{1}, y=\rho \omega$ with $r, \rho>0, \omega \in S^{n-1}$ in polar coordinates to obtain

$$
\left\|\left.\mathbf{f}\right|_{B_{R}}\right\|_{C^{0, \alpha-1}\left(B_{R} ; \mathbb{R}^{n}\right)}=\sup _{0<|x|<|y|<R} \frac{|\mathbf{f}(x)-\mathbf{f}(y)|}{|x-y|^{\alpha-1}}=\sup _{\substack{0<r<\rho<R \\ \omega \in S^{n-1}}} \frac{\left|\mathbf{f}\left(r e_{1}\right)-\mathbf{f}(\rho \omega)\right|}{\left|r e_{1}-\rho \omega\right|^{\alpha-1}}
$$

since $\mathbf{f}$ is continuous and $\mathbf{f}(P x)=P \mathbf{f}(x)$ for any orthogonal transformation $P$ on $\mathbb{R}^{n}$. We distinguish three cases according to $r$ and $\rho$ in the supremum. Note that $\left|\omega-t e_{1}\right|=\left(1+t^{2}-\right.$ $\left.2 t \omega_{1}\right)^{1 / 2}$ for $-1 \leq t \leq 1, \omega=\left(\omega_{1}, \omega_{2}, \ldots, \omega_{n}\right) \in S^{n-1}$. 
If $0<r<\rho \leq 1$, then Propositions B.3 and B.4 give

$$
\begin{aligned}
\frac{\left|\mathbf{f}\left(r e_{1}\right)-\mathbf{f}(\rho \omega)\right|}{\left|r e_{1}-\rho \omega\right|^{\alpha-1}} & =\frac{\left|r^{\alpha-1} e_{1}-\rho^{\alpha-1} \omega\right|}{\left|r e_{1}-\rho \omega\right|^{\alpha-1}} \\
& =\left(\Phi_{r / \rho,(r / \rho)^{\alpha-1}}\left(\omega_{1}\right)\right)^{1 / 2} \leq\left(\Phi_{r / \rho,(r / \rho)^{\alpha-1}}(-1)\right)^{1 / 2} \\
& =g_{\alpha}\left(\frac{r}{\rho}\right) \leq 2^{2-\alpha}
\end{aligned}
$$

If $1<r<\rho \leq R$, then Propositions B.3 and B.4 give

$$
\begin{aligned}
\frac{\left|\mathbf{f}\left(r e_{1}\right)-\mathbf{f}(\rho \omega)\right|}{\left|r e_{1}-\rho \omega\right|^{\alpha-1}} & =\frac{\left|e_{1} / r-\omega / \rho\right|}{\left|r e_{1}-\rho \omega\right|^{\alpha-1}} \\
& =\frac{1}{\rho^{\alpha}}\left(\Phi_{r / \rho, \rho / r}\left(\omega_{1}\right)\right)^{1 / 2} \leq \frac{1}{\rho^{\alpha}}\left(\Phi_{r / \rho, \rho / r}(-1)\right)^{1 / 2} \\
& =\frac{1}{\rho^{\alpha}} \tilde{g}_{\alpha}\left(\frac{r}{\rho}\right) \leq \frac{1}{\rho^{\alpha}} \widetilde{g}_{\alpha}\left(\frac{1}{\rho}\right)=\tilde{g}_{\alpha}(\rho) \leq 2^{2-\alpha}
\end{aligned}
$$

If $0<r \leq 1<\rho \leq R$, then Propositions B.3 and B.4 give

$$
\begin{aligned}
\frac{\left|\mathbf{f}\left(r e_{1}\right)-\mathbf{f}(\rho \omega)\right|}{\left|r e_{1}-\rho \omega\right|^{\alpha-1}} & =\frac{\left|r^{\alpha-1} e_{1}-\omega / \rho\right|}{\left|r e_{1}-\rho \omega\right|^{\alpha-1}} \\
& =\frac{1}{\rho^{\alpha}}\left(\Phi_{r / \rho, r^{\alpha-1} \rho}\left(\omega_{1}\right)\right)^{1 / 2} \leq \frac{1}{\rho^{\alpha}}\left(\Phi_{r / \rho, r^{\alpha-1} \rho}(-1)\right)^{1 / 2} \\
& =G_{\alpha, r}(\rho) \leq 2^{2-\alpha} .
\end{aligned}
$$

The proof is now completed.

\section{Acknowledgments}

Kei Morii is supported by the JSPS Global COE Program, Kyoto University. Yoshihiro Sawano was supported by Grant-in-Aid for Young Scientists (B) (no. 21740104) Japan Society for the Promotion of Science. Hidemitsu Wadade is supported by the Fujukai Foundation.

\section{References}

[1] H. Brézis and T. Gallouët, "Nonlinear Schrödinger evolution equations," Nonlinear Analysis, vol. 4, no. 4, pp. 677-681, 1980.

[2] H. Brézis and S. Wainger, "A note on limiting cases of Sobolev embeddings and convolution inequalities," Communications in Partial Differential Equations, vol. 5, no. 7, pp. 773-789, 1980.

[3] T. Ozawa, "On critical cases of Sobolev's inequalities," Journal of Functional Analysis, vol. 127, no. 2, pp. 259-269, 1995. 
[4] H. Kozono, T. Ogawa, and Y. Taniuchi, "The critical Sobolev inequalities in Besov spaces and regularity criterion to some semi-linear evolution equations," Mathematische Zeitschrift, vol. 242, no. 2, pp. 251-278, 2002.

[5] T. Ogawa, "Sharp Sobolev inequality of logarithmic type and the limiting regularity condition to the harmonic heat flow," SIAM Journal on Mathematical Analysis, vol. 34, no. 6, p. 1318-1330 (electronic), 2003.

[6] Y. Sawano, "Brézis-Gallouët-Wainger type inequality for Besov-Morrey spaces," Studia Mathematica, vol. 196, no. 1, pp. 91-101, 2010.

[7] K. Morii, T. Sato, and Y. Sawano, "Certain identities on derivatives of radial homogeneous and logarithmic functions," Communications in Mathematical Analysis, vol. 9, no. 2, pp. 51-66, 2010.

[8] K. Morii, T. Sato, and H. Wadade, "Brézis-Gallouët-Wainger type inequality with a double logarithmic term in the Hölder space: its sharp constants and extremal functions," Nonlinear Analysis, Theory, Methods and Applications, vol. 73, no. 6, pp. 1747-1766, 2010.

[9] H. Triebel, Theory of Function Spaces, vol. 78 of Monographs in Mathematics, Birkhäuser, Basel, Switzerland, 1983.

[10] H. Triebel, Theory of Function Spaces. III, vol. 100 of Monographs in Mathematics, Birkhäuser, Basel, Switzerland, 2006.

[11] R. A. Adams, Sobolev Spaces, vol. 6, Academic Press, New York, NY, USA, 1975.

[12] V.S. Rychkov, "On restrictions and extensions of the Besov and Triebel-Lizorkin spaces with respect to Lipschitz domains," Journal of the London Mathematical Society. Second Series, vol. 60, no. 1, pp. 237-257, 1999.

[13] L. C. Evans and R. F. Gariepy, Measure Theory and Fine Properties of Functions, Studies in Advanced Mathematics, CRC Press, Boca Raton, Fla, USA, 1992.

[14] M. H. Taibleson, “On the theory of Lipschitz spaces of distributions on Euclidean $n$-space. I. Principal properties," vol. 13, pp. 407-479, 1964.

[15] A. Lunardi, Analytic Semigroups and Optimal Regularity in Parabolic Problems, vol. 16 of Progress in Nonlinear Differential Equations and their Applications, Birkhäuser, Basel, Switzerland, 1995.

[16] S. Ibrahim, M. Majdoub, and N. Masmoudi, "Double logarithmic inequality with a sharp constant," Proceedings of the American Mathematical Society, vol. 135, no. 1, pp. 87-97, 2007. 